\title{
A Needed Rewriting Strategy for Data-Structures with Pointers ${ }^{\star}$
}

\author{
Rachid Echahed and Nicolas Peltier \\ LIG, CNRS \\ 46, avenue Félix Viallet \\ 38031 Grenoble Cedex, France \\ Rachid.Echahed@imag.fr, Nicolas.Peltier@imag.fr
}

\begin{abstract}
We propose a reduction strategy for systems of rewrite rules operating on term-graphs. These term-graphs are intended to encode pointer-based data-structures that are commonly used in programming, with cycles and sharing. We show that this reduction strategy is optimal w.r.t. a given dependency schema, which intuitively encodes the "interferences" among the nodes in the term-graph. We provide a new way of computing such dependency schemata.
\end{abstract}

\section{Introduction}

It is well-known that term-graph rewrite systems are non confluent in general, even if we restrict ourselves to standard orthogonal systems. A system as simple as $f(x) \rightarrow x, g(x) \rightarrow x$ is non confluent when applied on a cyclic term-graph $\alpha=f(g(\alpha)$ (two distinct normal forms exist: $\alpha=f(\alpha)$ or $\alpha=g(\alpha)$ ). Things get even worse if the rules are allowed to "physically" affect the term-graph by relabeling some of its nodes or by redirecting some edges occurring in it, because in this case two distinct functions may modify or access to the same nodes, making the result obviously dependent on the evaluation ordering. Assume for instance that we write a function insert that physically inserts an element at the end of a list. We can call this function on the list $\alpha=[1,2,3]$ and on the element length $(\alpha)$, where $\alpha$ points to the same (physical) list $[1,2,3]$. According to the order in which the two functions length and insert are evaluated, we can obtain either $[1,2,3,3]$ (if length is evaluated first, then the result inserted in the list) or $[1,2,3,4]$ if we proceed in a "lazy" way, by inserting the element before computing its value.

Obviously, functions of this kind, operating on pointer-based data-structures, are ubiquous in programming, because they allow the programmer to avoid duplication of information, hence to reduce the amount of needed memory. For instance, redirections are needed if one wants to define in-place algorithms for reversing or sorting a list. Another well-known example is the Shorr-Waite algorithm [11] which uses a link reversal technique to avoid the need for a stack during the exploration of a graph.

\footnotetext{
* This work has been partly funded by the project ARROWS of the French Agence
} Nationale de la Recherche. 
In [7] we proposed a solution to this problem (for constructor-based rewrite rules). The idea is to fix a specific evaluation ordering, by assuming a given priority ordering (denoted by $\preceq$ ) among the nodes. Then the "strict" rewriting strategy merely consists in reducing systematically the maximal node (according to $\preceq$ ). This strategy is deterministic (if the rewrite system is orthogonal) thus trivially confluent. Of course it is not satisfactory since it may be inefficient (it can be compared to purely imperative programs, or to a kind of "innermost" rewriting where the priority ordering replaces the subterm relation). It is only useful as a way to define the semantics of the term-graph rewrite systems (namely the expected normal forms), but should not be used in practice to compute the values. Therefore we defined more flexible reduction strategies that are allowed to reduce nodes that are non maximal w.r.t. $\succeq$ but only under some particular conditions which are strong enough to ensure that confluence is preserved.

In order to define these conditions, we introduced the notion of dependency schema, which is a set of relations specifying, in some sense, which nodes interfere with a given node in a term-graph (i.e., given a node $\alpha$ in a term-graph, which nodes affect $\alpha$ and which nodes depend on $\alpha$ ). We proved confluence of the flexible strategy for a class of rewrite systems. A dependency schema needs not to be provided by the user: we presented an algorithm to automatically compute a dependency schema having the desired properties.

In the present paper we extend our results on two aspects. First, we introduce a refined dependency schema, which is more powerful than the previous one, in the sense that it provides a more precise approximation of the "ideal" interference relation (which is non computable). Second, we propose a particular rewrite strategy consisting in computing only the nodes that are - in some sense relevant for the considered term-graph. We show that this strategy is normalizing (i.e. all the normal forms can be reached). Moreover, for a restricted class of term-graph rewrite systems, this strategy is optimal, in the sense that it only rewrites nodes that are really needed to obtain the normal form (i.e. they are reduced in any derivation leading to the considered normal form). It should be mentioned that optimality is defined here w.r.t. a particular dependency schema (optimality in general is trivially impossible). The class of rules we consider is not too restrictive because every inductively/strongly sequential rewrite system (in the standard sense $[9,10,8]$ ) can be reduced to it.

Our work extends existing results for term rewrite systems [1] and graph rewrite systems $[4,5]$ for which optimal rewriting strategies are known since a long time ago (for inductively/strongly sequential systems). We hope that it provides a good theoretical basis for defining a programming language based on term-graphs, that will offer similar features as rewrite-based languages such as Haskell [12] (namely efficient, lazy reduction strategies) and in the same time will be sufficiently expressive to allow the programmer to fully control the allocation of memory when (and if) needed.

The rest of the paper is organized as follows. Section 2 defines the notion of term-graphs and actions operating on them. Section 3 introduces the notion of 
graph rewrite rules, dependency schemata, and defines rewriting relations based on them. We prove that the flexible rewriting relation is confluent for orthogonal systems. The notions we use are slightly different (and much simpler than) from the ones in [7], but the previous results are essentially the same ${ }^{1}$. Section 4 provides an example of a dependency schema, distinct from (and strictly more powerful than) the one already proposed in [7]. Section 5 is the hearth of the paper: it contains the main new result, namely a reduction strategy which is optimal for a particular class of rules (called elementary). Section 6 provides simple examples of applications.

\section{Basic Definitions}

\subsection{Term-Graphs}

We use the word "term-graph" to denote data-structures defined by a set of nodes connected by labeled directed edges. Edges are assumed to be unique, i.e. given a node $\alpha$ and a label $a$, there can be at most one edge starting from $\alpha$ and labeled by $a$.

Formally, we assume given a set of nodes $\mathcal{N}$, denoted by Greek letters, and a set of features $\mathcal{F}$, denoted by $a, b, \ldots$ Features may be seen as (partial) functions from $\mathcal{N}$ to $\mathcal{N}$, or as edge labels. $\mathcal{F}$ contains at least a special element $l$, which will be used to denote the label (or head symbol) of the node $\alpha$. We also assume given a total ordering $\succeq$ on $\mathcal{N}$, called the priority ordering, and specifying the order in which the nodes should be reduced (as explained in the Introduction).

Let $\mathcal{P}$ be a subset of $\mathcal{N}$, called the set of predefined nodes. We use predefined nodes mainly to encode function symbols: $\mathcal{P}$ contains a set of functions $\Sigma$ (denoted by $f, g, h, \ldots)$ divided into two disjoint sets of symbols: a set $\mathcal{D}$ of defined symbols and a set $\mathcal{C}$ of constructors. The value of the feature $l$ of a node $\alpha$ gives the label (i.e. head symbol) of $\alpha$. Predefined nodes could also be used to denote built-in values such as reals or integers.

Definition 1. A term-graph $t$ is defined by:

- A set of nodes $\mathcal{N}(t) \subseteq \mathcal{N} \backslash \mathcal{P}$.

- A function mapping each symbol a in $\mathcal{F}$ to a partial function $a_{t}$ from $\mathcal{N}(t)$ to $\mathcal{N}(t) \cup \mathcal{P}$. If $a_{t}(\alpha)=\beta$ then we say that $t$ contains an edge from $\alpha$ to $\beta$, labeled by $a$, or that the feature $a$ of $\alpha$ is $\beta$. We assume that $l_{t}(\alpha) \in \Sigma$ if $l_{t}(\alpha)$ is defined.

$A$ rooted term-graph is a term-graph associated with a distinguished node $\alpha$, called the root of $t$ and denoted by $\operatorname{root}(t)$.

\footnotetext{
1 The differences are mainly due to the fact that in contrast to [7] we make no distinction between actions affecting the name of a node, and actions only redirecting its edges. Consequently, the reduction strategies are less flexible, but on the other hand this yields a much simpler and more intuitive framework. Moreover we also slightly modify some of the definitions in order to properly handle non orthogonal rewrite systems.
} 
For instance, a term $f(a, x)$ (where $x$ is a variable) is represented by a termgraph $t$ of root $\alpha$ s.t. $\mathcal{N}(t)=\{\alpha, \beta, \delta\}, l_{t}(\alpha)=f, l_{t}(\beta)=a, 1_{t}(\alpha)=\beta, 2_{t}(\alpha)=\delta$. The feature $i$ where $i \in \mathbb{N}$ denotes the $i$-th argument of a function.

We denote by $\operatorname{dom}(t)$ the set of nodes $\alpha$ s.t. $a_{t}(\alpha)$ is defined for at least one feature $a$ (the other nodes can be seen as variables).

Let $t, s$ be two term-graphs. We write $t \subseteq s$ iff $t$ is included in $s$ i.e. iff $\mathcal{N}(t) \subseteq \mathcal{N}(s)$ and $a_{t}(\alpha)=a_{s}(\alpha)$, for every node $\alpha$ s.t. $a_{t}(\alpha)$ is defined.

\section{$2.2 \mathcal{N}$-Mappings}

An $\mathcal{N}$-mapping is a total function from $\mathcal{N}$ to $\mathcal{N}$ s.t. for every $\alpha \in \mathcal{P}$ and for every $\beta \in \mathcal{N}, \sigma(\beta)=\alpha \Leftrightarrow \alpha=\beta$ (predefined nodes are left unchanged and no non predefined node can be mapped to a predefined node).

An $\mathcal{N}$-mapping $\sigma$ is said to be compatible with a term-graph $t$ if for all $\alpha, \beta \in \mathcal{N}(t)$ s.t. $\sigma(\alpha)=\sigma(\beta)$ and for all $a \in \mathcal{F}$, if $a_{t}(\alpha)$ and $a_{t}(\beta)$ are defined, then $\sigma\left(a_{t}(\alpha)\right)=\sigma\left(a_{t}(\beta)\right)$.

In this case, $\sigma(t)$ denotes the term-graph $s$ defined as follows: $\mathcal{N}(s) \stackrel{\text { def }}{=}\{\sigma(\alpha) \mid$ $\alpha \in \mathcal{N}(t)\}$, and $a_{s}(\sigma(\alpha)) \stackrel{\text { def }}{=} \sigma\left(a_{t}(\alpha)\right)$.

Example 1. Let $t$ be the term-graph defined by $\mathcal{N}(t)=\{\alpha, \beta, \gamma, \delta, \lambda\}, l_{t}(\alpha)=f$, $l_{t}(\beta)=l_{t}(\gamma)=g, 1_{t}(\alpha)=\beta, 2_{t}(\alpha)=\gamma, 1_{t}(\beta)=\delta, 1_{t}(\gamma)=\lambda$ (denoting the term $f(g(\delta), g(\lambda)))$. Then the function $\sigma$ s.t. $\sigma(\beta)=\sigma(\gamma)=\sigma(\delta)=\lambda, \sigma(\alpha)=\alpha$ and $\sigma(\lambda)=\lambda$ is compatible with $t$. On the other hand, we cannot take $\sigma(\beta)=\sigma(\alpha)$, because $l_{t}(\alpha)=f$ and $l_{t}(\beta)=g$, and $\sigma(f)=f \neq \sigma(g)=g$.

An $\mathcal{N}$-mapping $\sigma$ is said to be a renaming if it is injective and $\sigma(\alpha) \succ \sigma(\beta)$ for any $\alpha \succ \beta$. Note that by definition a renaming is compatible with any termgraph $t$.

An $\mathcal{N}$-relation $\Delta$ is a relation on the nodes of a term-graph which is independent from the names of the nodes. Formally, it is a function mapping every term-graph $t$ to a relation $\Delta_{t}$ on the nodes of $t$ s.t. for every renaming $\eta$ and for every pair of nodes $\alpha, \beta$ occurring in $t$ we have $\alpha \Delta_{t} \beta$ iff $\eta(\alpha) \Delta_{\eta(t)} \eta(\beta)$.

One of the simplest examples of an $\mathcal{N}$-relation is the relation $\geq_{t}$ defined as the smallest reflexive and transitive relation s.t. for all term-graphs $t$, for all $a \in \mathcal{F}$ and for all $\alpha \in \operatorname{dom}\left(a_{t}\right)$, we have $\alpha \geq_{t} a_{t}(\alpha)\left(\alpha \geq_{t} \beta\right.$ iff there is a path from $\alpha$ to $\beta$ in $t$ ).

\subsection{Actions}

The definitions of actions and rewrite rules are close to the ones of $[6,7]$, but slightly simpler. An action is one of the following forms:

- an edge redirection/creation $\alpha \gg_{a} \beta$ where $\alpha, \beta$ are nodes, $a$ is a feature and $\alpha \notin \mathcal{P}$. This means that the value of $a(\alpha)$ is changed to $\beta$. This may be seen as an edge redirection: the target of the edge starting from $\alpha$ and labeled by $a$ is redirected to point to $\beta$. The edge and nodes are created if they do not exist. If $a=l$ then we assume that $\beta \in \Sigma$. 
- a global redirection $\alpha \gg \beta$ where $\alpha$ and $\beta$ are nodes and $\alpha \notin \mathcal{P}$. This means that all edges pointing to $\alpha$ are redirected to $\beta$.

Note that predefined nodes cannot be redirected. The result of applying an action $\epsilon$ to a term-graph $t$ is denoted by $\epsilon[t]$ and is defined as the term-graph $s$ s.t.:

- If $\epsilon=\alpha \gg_{a} \beta$ then $\mathcal{N}(s) \stackrel{\text { def }}{=} \mathcal{N}(t) \cup\{\alpha, \beta\}, a_{s}(\alpha) \stackrel{\text { def }}{=} \beta$ and for all features $b$ and all nodes $\gamma$ we have $b_{s}(\gamma) \stackrel{\text { def }}{=} b_{t}(\gamma)$ iff $a \neq b$ or $\gamma \neq \alpha$.

- If $\epsilon=\alpha \gg \beta$ then $\mathcal{N}(s) \stackrel{\text { def }}{=} \mathcal{N}(t) \cup\{\alpha, \beta\}$ and for all features $a$ and all nodes $\gamma, a_{s}(\gamma) \stackrel{\text { def }}{=} \beta$ if $a_{t}(\gamma)=\alpha$ and $a_{s}(\gamma) \stackrel{\text { def }}{=} a_{t}(\gamma)$ otherwise.

If $\varsigma$ is a finite sequence of actions, then $\varsigma[t]$ is defined inductively as follows: $\varsigma[t] \stackrel{\text { def }}{=} t$ if $\varsigma$ is empty and $(\epsilon ; \varsigma)[t] \stackrel{\text { def }}{=} \varsigma[\epsilon[t]]$ (where $\epsilon$ is an action and; denotes the concatenation operator). $\mathcal{N}(\varsigma)$ denotes the set of nodes occurring in $\varsigma$. $\operatorname{dom}(\varsigma)$ denotes the set of nodes $\alpha$ s.t. $\varsigma$ contains an action of the form $\alpha \gg \beta$ or $\alpha \gg_{a} \beta$.

Example 2. The following sequence of actions inserts the element 0 at second position in a list. We assume that lists are labeled by cons, the feature car gives the first element and $c d r$ the tail. Initially, the list is $\alpha$ and its tail is $\beta$ : $\alpha \gg_{c d r} \delta ; \delta \gg_{l}$ cons; $\delta \gg_{c a r} \gamma ; \delta \gg_{c d r} \beta ; \gamma \gg_{l} 0$.

Proposition 1. Let $\varsigma, \varsigma^{\prime}$ be two sequences of actions. If $\operatorname{dom}(\varsigma) \cap \mathcal{N}\left(\varsigma^{\prime}\right)=$ $\operatorname{dom}\left(\varsigma^{\prime}\right) \cap \mathcal{N}(\varsigma)=\emptyset$, then $\varsigma^{\prime}[\varsigma[t]]=\varsigma\left[\varsigma^{\prime}[t]\right]$.

Proof. Let $\varsigma=\epsilon_{1} \ldots \epsilon_{n}$ and $\varsigma^{\prime}=\epsilon_{1}^{\prime} \ldots \epsilon_{m}^{\prime}$. The proof is by induction on the pair $(m, n)$.

If $n=0$ or $m=0$ the proof is immediate.

Assume that $m=n=1$. Let $s=\left(\varsigma ; \varsigma^{\prime}\right)[t]$ and $s^{\prime}=\left(\varsigma^{\prime} ; \varsigma\right)[t]$.

$\varsigma, \varsigma^{\prime}$ are respectively of the form $\alpha \gg_{\star} \beta$ and $\alpha^{\prime} \gg_{\bullet} \beta^{\prime}$ where $\star, \bullet$ are either features in $\mathcal{F}$ or empty (in case of global redirections). Since $\operatorname{dom}(\varsigma) \cap \mathcal{N}\left(\varsigma^{\prime}\right)=\emptyset$ we have $\alpha \neq \alpha^{\prime}, \beta^{\prime}$ and since $\operatorname{dom}\left(\varsigma^{\prime}\right) \cap \mathcal{N}(\varsigma)=\emptyset$, we have $\alpha^{\prime} \neq \alpha, \beta$.

We have $\mathcal{N}(s) \stackrel{\text { def }}{=} \mathcal{N}(t) \cup\{\alpha, \beta\} \cup\left\{a l p h a^{\prime}, \beta^{\prime}\right\}=\left\{\alpha, \beta, \alpha^{\prime}, \beta^{\prime}\right\}$. Thus by symmetry $\mathcal{N}(s)=\mathcal{N}\left(s^{\prime}\right)$.

Now, assume that there exists a node $\lambda$ and a feature $c$ s.t. $c_{s}(\lambda) \neq c_{s^{\prime}}(\lambda)$.

If $\star=c$ and $\alpha=\lambda$, then we must have $c_{\varsigma[t]}(\lambda)=\beta$. Since $\alpha^{\prime} \neq \beta$ and $\alpha^{\prime} \neq \alpha$, neither $\alpha$ nor $\beta$ can be redirected by $\varsigma^{\prime}$, thus $c_{s}(\lambda)=\beta$. Moreover, we have by definition $c_{s^{\prime}}(\lambda)=\beta$.

By symmetry, we assume that $\bullet \neq c$ or $\alpha^{\prime} \neq \lambda$.

If $\star$ is empty and $\alpha=c_{t}(\lambda)$ then $c_{\varsigma[t]}(\lambda)=\beta$. We have $\alpha^{\prime} \neq \beta$. Moreover if $\bullet=c$ then $\alpha^{\prime} \neq \lambda$. Thus $c_{s}(\lambda)=\beta$. Moreover $c_{t\left[\varsigma^{\prime}\right]}(\lambda)=\alpha$, hence $c_{s^{\prime}}(\lambda)=\beta$.

By symmetry we assume that either $\bullet$ is not empty or that $\alpha^{\prime} \neq c_{t}(\lambda)$.

Thus, neither $\varsigma$ nor $\varsigma^{\prime}$ can change the value of $c_{t}(\lambda)$, hence $c_{s}(\lambda)=c_{s^{\prime}}(\lambda)$.

If $n, m>1$, then $\varsigma^{\prime}[\varsigma[t]]=\epsilon_{1} ; \ldots ; \epsilon_{n} ; \epsilon_{1}^{\prime} ; \ldots ; \epsilon_{m}^{\prime}[t]=$ $\epsilon_{1}^{\prime} ; \ldots ; \epsilon_{m-1}^{\prime} ; \epsilon_{1} ; \ldots ; \epsilon_{n} ; \epsilon_{m}^{\prime}[t]$ (by the induction hypothesis, applied with $m-1)$. We can apply the induction hypothesis on $(n, 1)$. We get: $\epsilon_{1}^{\prime} ; \ldots ; \epsilon_{m-1}^{\prime} ; \epsilon_{1} ; \ldots ; \epsilon_{n} ; \epsilon_{m}^{\prime}[t]=\epsilon_{1}^{\prime} ; \ldots ; \epsilon_{m}^{\prime} ; \epsilon_{1} ; \ldots ; \epsilon_{n}[t]$, hence the proof is completed. 


\subsection{A Linear Notation for Actions and Term-Graphs}

For the sake of conciseness and readability, we introduce another notation for denoting sequences of actions. They will be denoted as terms with labels.

The term $\alpha: f\left(a_{1} \Rightarrow \beta_{1}: t_{1}, \ldots, a_{n} \Rightarrow \beta_{n}: t_{n}\right)$ will be used as a shortcoming for denoting the sequence of actions: $\left(\alpha \gg_{l} f\right) ;\left(\alpha \gg_{a_{1}} \beta_{1}\right) ; \ldots ;\left(\alpha \gg_{a_{n}}\right.$ $\left.\beta_{n}\right) ; \tau_{1} ; \ldots ; \tau_{n}$ where $\tau_{1}, \ldots, \tau_{n}$ are the sequences of actions corresponding to the terms $\beta_{1}: t_{1}, \ldots, \beta_{n}: t_{n}$, respectively. For instance, $\alpha: \operatorname{cons}(\operatorname{car} \Rightarrow \beta: 0, c d r \Rightarrow \gamma: n i l)$ denotes the sequence: $\alpha \gg_{l}$ cons; $\alpha \gg_{c a r} \beta ; \alpha \gg_{c d r} \gamma ; \beta \gg_{l} 0 ; \gamma \gg_{l}$ nil. The nodes $\alpha, \beta_{1}, \ldots, \beta_{n}$ can be left unspecified, and in this case they are simply replaced by arbitrary nodes not occurring elsewhere: for instance cons $(\operatorname{car} \Rightarrow 0)$

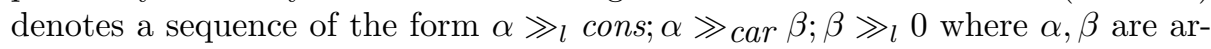
bitrarily chosen nodes. $f\left(t_{1}, \ldots, t_{n}\right)$ is syntactic sugar for $f\left(1 \Rightarrow t_{1}, \ldots, n \Rightarrow t_{n}\right)$ (where $1, \ldots, n \in \mathcal{F}$ ).

The same notation can be used to denote term-graphs. Indeed, a term-graph $t$ can be described by giving a sequence of actions $\varsigma$ s.t. $\varsigma[\emptyset]=t$, where $\emptyset$ denotes the empty graph (no nodes and no edges). For instance, $\alpha: a$, which denotes the action $\alpha \gg_{l} a$, will also denote a term-graph $t$ s.t. $\mathcal{N}(t)=\{\alpha\}$ where $l_{t}(\alpha)=a$ and $b_{t}(\alpha)$ is undefined if $b \neq l$. The term $\alpha$ denotes the term-graph reduced to the unique node $\alpha$, with no edges.

The operator ";", used to denote composition of actions, is also used to denote a union of term-graphs, for instance $\alpha: f(\delta) ; \beta: g(\delta)$ denotes a term-graph $t$ of nodes $\alpha, \beta, \delta$, s.t. $l_{t}(\alpha)=f, l_{t}(\beta)=g, 1_{t}(\alpha)=1_{t}(\beta)=\delta$. By convention, the root of the term-graph is the first mentioned node. For instance $\operatorname{root}(t)=\alpha$.

$\beta: g(\delta) ; \alpha: f(\delta)$ and $\delta ; \beta: g(\delta) ; \alpha: f(\delta)$ both denote the same term-graph, but with the roots $\beta$ and $\delta$ respectively (note that the term-graphs may contain nodes that are not reachable from the root).

\section{Term-Graph Rewriting}

Definition 2. (Rewrite Rule) $A$ term-graph rewrite rule is an expression of the form $L \rightarrow R \mid \phi$ where $L$ is a rooted term-graph, $R$ is a sequence of actions, and $\phi$ is a conjunction of disequations between nodes $\bigwedge_{i=1}^{n} \alpha_{i} \neq \beta_{i}$.

$A$ graph rewrite system (GRS for short) is a set of rewrite rules.

A rule is said to be admissible iff the following conditions hold:

- For every node $\alpha$ occurring in $L$, we have $\operatorname{root}(L) \geq_{L} \alpha$. Any node occurring in the left-hand side must be reachable from the root $^{2}$.

- We have $l_{L}(\operatorname{root}(L)) \in \mathcal{D}$ and for every node $\alpha \neq \operatorname{root}(L)$ if $l_{L}(\alpha)$ is defined then $l_{L}(\alpha) \in \mathcal{C}$. The root is the only node that is labeled by a non constructor symbol. This condition is usual in constructor-based rewrite systems.

\footnotetext{
${ }^{2}$ This is an important condition since otherwise the same rule could be applied in several different ways at the same node, which would make the system trivially non confluent in general.
} 
- For every action $\alpha \gg_{l} f$ in $R$ s.t. $f \in \mathcal{D}$, either $\alpha=\operatorname{root}(L)$ or $\alpha$ does not occur in $L$. Moreover if $\alpha \gg \beta \in R$ then $\beta \notin \Sigma$. Only the created (new) nodes are allowed to be labeled by a defined symbol ${ }^{3}$.

In the following we always assume that the rules are admissible.

In practice, rather than writing the right-hand side as a sequence of actions (which is not very convenient), we often prefer to use the linear notation in Section 2.4 which is clearer. For instance, we shall write $\alpha: a \rightarrow \alpha: f(a)$, instead of $\alpha: a \rightarrow \alpha \gg_{l} f ; \alpha \gg_{1} \beta ; \beta \gg_{l} a$.

If this notation is used, then we always implicitly assume that the root of the left-hand side is redirected to the root of the term-graph denoted by the right-hand side. For instance, $\alpha: f(a) \rightarrow \beta: g(b)$ denotes the rule: $\alpha: f(a) \rightarrow \alpha \gg$ $\beta ; \beta \gg_{l} g ; \beta \gg_{1} \gamma ; \gamma \gg_{l} b$ (the action $\alpha \gg \beta$ is added at the beginning of the sequence). Using these conventions any term rewrite rule (in the usual sense) can be seen as a term-graph rewrite rule. These conventions (as the ones in Section 2.4) are only introduced as syntactic sugar to make the notations and examples clearer and easier to understand. They do not affect the semantics.

A substitution $(\mathcal{N}$-mapping $) \sigma$ is said to be a solution of a conjunction of disequations $\phi=\bigwedge_{i=1}^{n}\left(\alpha_{i} \neq \beta_{i}\right)$ iff for every $i \in[1 . . n], \sigma\left(\alpha_{i}\right) \neq \sigma\left(\beta_{i}\right)$. The set of solutions of $\phi$ is denoted by $\operatorname{sol}(\phi)$.

Definition 3. Let $\rho: L \rightarrow R \mid \phi$ be a rule. A $\rho$-matcher for a term-graph $t$ (at a node $\alpha \in \mathcal{N}(t)$ ) is an $\mathcal{N}$-mapping $\sigma$ compatible with $L$ satisfying the following conditions.

1. $\sigma(L) \subseteq t$, i.e. $\sigma(L)$ must be a subgraph of $t$.

2. $\sigma \in \operatorname{sol}(\phi)$ and $\alpha=\sigma(\operatorname{root}(L))$.

3. Let $N$ be the set of nodes occurring in $R$ but not in $L . N$ corresponds to nodes that are created by the rewrite rule. $\sigma$ maps the nodes in $N$ to pairwise distinct nodes not occurring in $t$ s.t.:

- If $\beta, \gamma$ are two nodes in $N$ s.t. $\beta \succeq \gamma$ then $\sigma(\beta) \succeq \sigma(\gamma)$. This means that the newly created nodes should be ordered as specified in the rewrite rule.

- For every node $\beta$ occurring in $t$, and for every node $\gamma$ in $N, \beta \prec \sigma(\gamma)$ iff $\beta \preceq \alpha$. This means that the newly created nodes inherit the priority of the parent node $\alpha$.

$\rho$-matchers can be easily computed using standard matching algorithms. If $\sigma$ is a $\rho$-matcher for $t$, where $\rho=L \rightarrow R \mid \phi$, then we denote by $\rho^{\sigma}[t]$ the term-graph $\sigma(R)[t]$ obtained by applying the sequence of actions $\sigma(R)$ on $t$.

Let $\mathcal{R}$ be a GRS. Let $t$ be a term-graph. We define the following relations between nodes (implicitly depending on $\mathcal{R}$ ):

$-\alpha \triangleright_{t} \beta$ iff there is a rule $\rho: L \rightarrow R \mid \phi \in \mathcal{R}$ and a $\rho$-matcher $\sigma$ s.t. $\beta \in \sigma(L)$.

This means that a rule that depends on the node $\beta$ is applicable on $\alpha$

\footnotetext{
3 This condition is not really restrictive since one can easily redirect an existing node $\alpha$ to a new node $\beta$ before relabeling it.
} 
$-\alpha \gg_{t} \beta$ iff there is a rule $\rho: L \rightarrow R \mid \phi \in \mathcal{R}$ and a $\rho$-matcher $\sigma$ at $\alpha$ s.t. $\beta \in \operatorname{dom}(\sigma(R))$. This means that a rule affecting $\beta$ can be applied on $\alpha$.

Example 3. Assume that $\mathcal{R}=\{f(\alpha: 0, \beta: s(\delta)) \rightarrow \beta: 2\}$. Let $t=$ $\lambda_{1}: f\left(\lambda_{2}: 0, \lambda_{3}: s\left(\lambda_{4}: s\left(\lambda_{5}: 0\right)\right)\right)$. Then we have $\lambda_{1} \triangleright_{t} \lambda_{1}, \lambda_{2}, \lambda_{3}, \lambda_{4}$ and $\lambda_{2} \triangleright_{t} \lambda_{5}$. We have $\lambda_{1} \gg_{t} \lambda_{1}, \lambda_{3}$ and $\lambda_{1} \ngtr_{t} \lambda_{2}, \lambda_{4}, \lambda_{5}$.

Definition 4. Let $\mathcal{R}$ be a set of rewrite rules. An $\mathcal{R}$-dependency schema is a pair $\left(\gg^{*}, \triangleright^{*}\right)$ of $\mathcal{N}$-relations s.t. for every term-graph $t, \gg_{t}^{*}, \triangleright_{t}^{*}$ contain $\gg_{t}$ and $\triangleright_{t}$ respectively.

Let $\rho=L \rightarrow R \mid \phi$ be a rule and let $\sigma$ be a $\rho$-matcher for a term-graph $t$ at $\alpha$. We write $\sigma \bowtie_{t}^{\xi} \beta$ if $\alpha \prec \beta$ and there exists a node $\gamma$ s.t. either $\gamma \in \operatorname{dom}(\sigma(R))$ and $\beta \triangleright_{t}^{*} \gamma$ or $\gamma \in \mathcal{N}(\sigma(L))$ and $\beta \gg_{t}^{*} \gamma$.

Informally, the intended meaning of $\alpha \gg_{t}^{*} \gamma$ is that the reduction of $\alpha$ may affect the node $\gamma$, possibly after some reduction steps. $\alpha \triangleright_{t}^{*} \gamma$ means that the value of the node $\alpha$ may depend on the value of $\gamma$. For both relations, reduction must be taken into account (see Definition 5). For instance, if length is the function defined as usual on lists, and $t$ is the term $\alpha:$ length $(\operatorname{cons}(0, \operatorname{cons}(1, \operatorname{cons}(2, \beta))))$, then we have $\alpha \not_{t} \beta$, but $\alpha \triangleright_{t}^{*} \beta$ (the value of $\alpha$ depends on $\beta$, but only after some reduction steps).

$\sigma \bowtie_{t}^{\xi} \beta$ expresses the fact that there is a potential "conflict" between the rule $\rho$ corresponding to $\sigma$ and the node $\beta$ (according to the considered dependency schema): either $\rho$ redirects a node $\gamma$ on which $\beta$ (possibly) depends, or $\rho$ uses a node $\gamma$ which may be affected by $\beta$. From an intuitive point of view, $\gamma$ may be seen as a resource that is shared between $\rho$ and $\beta$.

Let $\mathcal{R}$ be a rewrite system and let $\xi$ be an $\mathcal{R}$-dependency schema. A matcher $\sigma$ for $t$ at a node $\alpha$ is said to be eligible if there is no node $\beta$ in $t$ s.t. $\sigma \bowtie_{t}^{\xi} \beta$. We define the three following rewriting relations.

- $t \rightarrow_{\mathcal{R}} s$ iff there exist a rule $\rho \in \mathcal{R}$ and a $\rho$-matcher $\sigma$ for $t$ s.t. $s=\rho^{\sigma}[t]$. This is the basic rewriting relation, close in spirit to the one used for terms. It is non confluent even if $\mathcal{R}$ is orthogonal [3] and even if all nodes only affect themselves.

$-t \succ_{\mathcal{R}} s$ iff there exist a rule $\rho \in \mathcal{R}$ and a $\rho$-matcher $\sigma$ for $t$ at a node $\alpha$ s.t. $s=\rho^{\sigma}[t]$ and if for every node $\beta$ in $t$, we have $\alpha \succeq \beta$ or $l_{t}(\beta) \in \mathcal{C}$. This means that the rules are applied only on maximal reducible nodes (according to the ordering $\succeq$ ). This relation is called strict rewriting. It is deterministic and one can view strict rewrite systems as purely imperative programs, in the sense that the order in which the actions are performed is entirely specified.

$-t \stackrel{\xi}{\rightarrow}_{\mathcal{R}} s$ iff there exist a rule $\rho \in \mathcal{R}$ and an eligible $\rho$-matcher $\sigma$ for $t$ at a node $\alpha$ s.t. $s=\rho^{\sigma}[t] . \stackrel{\xi}{\rightarrow}_{\mathcal{R}}$ is a rewriting relation that is more flexible than $\breve{\zeta}_{\mathcal{R}}$ but as we shall see is also sufficiently strong to preserve confluence of orthogonal systems. The basic idea is that a rule $\rho$ may be applied on a non-maximal node $\alpha$ only if $\rho$ does not interfere with the reduction of the nodes $\beta \succ \alpha$. 


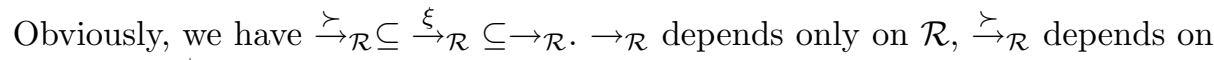
$\mathcal{R}$ and $\succ . \stackrel{\xi}{\rightarrow}_{\mathcal{R}}$ depends on $\mathcal{R}, \succ$ and $\xi$. If $\alpha \gg_{t}^{*} \beta$ and $\alpha \triangleright_{t}^{*} \beta$ for every pair of nodes $(\alpha, \beta)$ then $\stackrel{\xi}{\rightarrow}_{\mathcal{R}}$ coincides with $\stackrel{\succ}{\rightarrow}_{\mathcal{R}}$.

The following definition states additional semantic conditions on $\xi$ that are needed to ensure confluence.

Let $t, s$ be two term-graphs s.t. $t \stackrel{\xi}{\rightarrow}_{\mathcal{R}} s$. Let $\alpha$ be a node in $s$ and let $\beta$ be the node on which the rule is applied. We denote by $\alpha_{t \rightarrow s}^{-}$the node defined as follows: $\alpha_{t \rightarrow s}^{-} \stackrel{\text { def }}{=} \alpha$ if $\alpha \in \mathcal{N}(t)$ and $\alpha=\beta$ otherwise. $\alpha_{t \rightarrow s}^{-}$denotes the ancestor of the node $\alpha$.

Definition 5. An $\mathcal{N}$-relation $\Delta$ is said to be invariant for an $\mathcal{R}$-dependency schema $\xi$ if the following holds: if $\sigma$ is an eligible $\rho$-matcher for $t, s=\rho^{\sigma}[t]$ and $\beta \triangle_{s} \gamma$ then $\beta_{t \rightarrow s}^{-} \triangle_{t} \gamma_{t \rightarrow s}^{-}$. An $\mathcal{R}$-dependency schema $\xi=\left(\gg^{*}, \triangleright^{*}\right)$ is invariant if $\gg^{*}, \triangleright^{*}$ are invariant for $\xi$.

The intuitive idea is that one cannot discover new interferences during the derivation: if $\alpha, \beta$ are related at some point in the derivation, then either $\alpha, \beta$ or their ancestors were already in relation before.

\section{Examples}

We provide some simple examples of rewriting rules (defined using the above linear relation for the sake of conciseness and readability):

In situ append:

$a_{1}: \operatorname{append}(\alpha: n i l, \beta) \quad \rightarrow \beta ; \alpha \gg \beta \quad \% \alpha$ is redirected to $\beta$

$a_{2}$ : append $(\alpha: \operatorname{cons}(\beta, \delta), \gamma) \rightarrow \alpha ; \operatorname{append}(\delta, \gamma) \%$ Apply append on the tail

In situ increment of all the elements of a list:

$i_{1}: \operatorname{inc}(\alpha: n i l) \rightarrow \alpha \quad i_{2}: \operatorname{inc}(\alpha: \operatorname{cons}(\beta, \delta)) \rightarrow \alpha ; \alpha \gg_{1} s(\beta) ; \operatorname{inc}(\delta)$

In situ list reversal:

$r_{1}: \operatorname{rev}(\alpha) \rightarrow \beta: \operatorname{rev}^{\prime}(\alpha, n i l) ; \alpha \gg \beta \quad r_{2}: \operatorname{rev}^{\prime}(\alpha: n i l, \beta) \rightarrow \beta$

$r_{3}: \operatorname{rev}^{\prime}(\alpha: \operatorname{cons}(\beta, \delta), \gamma) \rightarrow \operatorname{rev}^{\prime}(\delta, \alpha) ; \alpha \gg_{2} \gamma$

Check whether an element occurs in a (possibly circular) list

$f_{1}: \operatorname{find}(\alpha, \beta) \rightarrow \lambda_{1}: \operatorname{find}^{\prime}(\alpha, \beta) ; \lambda_{2}: \operatorname{clean}(\alpha)$

$f_{2}: \operatorname{find}^{\prime}\left(\alpha, \beta\right.$ :cons $\left.\left(\alpha^{\prime}, \beta^{\prime}\right)\right) \rightarrow \operatorname{find}^{\prime}\left(\alpha, \beta^{\prime}\right) ; \beta \gg_{l}$ mark $\mid \alpha \neq \alpha^{\prime}$

$f_{3}:$ find $^{\prime}(\alpha, n i l) \rightarrow$ false $\quad f_{4}: f_{\text {find }}^{\prime}(\alpha, \operatorname{cons}(\alpha, \beta)) \rightarrow$ true

$f_{5}: \operatorname{find}\left(\alpha, \operatorname{mark}\left(\alpha^{\prime}, \beta\right)\right) \rightarrow$ false $f_{6}: \operatorname{clean}(\alpha: n i l) \rightarrow \alpha$

$f_{7}: \operatorname{clean}(\alpha: \operatorname{cons}(\beta, \delta)) \rightarrow \alpha \quad f_{8}: \operatorname{clean}(\alpha: \operatorname{mark}(\beta, \delta)) \rightarrow \operatorname{clean}(\delta) ; \alpha \gg_{l}$ cons

The function $f i n d^{\prime}(\alpha, \beta)$ explores the list until $\alpha$ is found. The nodes are marked in order to avoid looping. The function clean removes the marks. In this example, the ordering of the nodes in the right-hand side is crucial. We assume that $\lambda_{1} \succ \lambda_{2}$. Here are some examples of reductions $\left(\lambda_{1}, \lambda_{2}, \ldots\right.$ are new nodes created during the derivation):

$\operatorname{append}(\alpha: \operatorname{cons}(\beta, \delta: n i l), \alpha) \rightarrow a_{1} \alpha: \operatorname{cons}(\beta, \delta: n i l) ; \lambda_{1}: \operatorname{append}(\delta, \alpha) \rightarrow_{a_{2}} \alpha: \operatorname{cons}(\beta, \alpha)$ 


$$
\begin{aligned}
\operatorname{rev}\left(\alpha: \operatorname{cons}\left(\beta_{1}, \delta: \operatorname{cons}\left(\beta_{2}, n i l\right)\right)\right) & \rightarrow r_{1} \operatorname{rev}^{\prime}\left(\alpha: \operatorname{cons}\left(\beta_{1}, \delta: \operatorname{cons}\left(\beta_{2}, n i l\right)\right), \text { nil }\right) \\
& \rightarrow r_{3} \operatorname{rev}^{\prime}\left(\delta: \operatorname{cons}\left(\beta_{2}, n i l\right), \alpha: \operatorname{cons}\left(\beta_{1}, n i l\right)\right) \\
& \rightarrow r_{3} \operatorname{rev}\left(\operatorname{nil}, \delta: \operatorname{cons}\left(\beta_{2}, \alpha: \operatorname{cons}\left(\beta_{1}, n i l\right)\right)\right) \\
& \rightarrow r_{2} \delta: \operatorname{cons}\left(\beta_{2}, \alpha: \operatorname{cons}\left(\beta_{1}, n i l\right)\right)
\end{aligned}
$$

Confluence is an important property from a programming point of view, because it ensures that any object has a unique normal form (thus the defined symbols encode functions).

Lemma 1. Let $\mathcal{R}$ be a set of rewrite rules and let $\xi$ be an $\mathcal{R}$-dependency schema.

Let $\rho \in \mathcal{R}$. Let $t, s$ be two term-graphs and let $\eta$ be a renaming. Assume that $t \stackrel{\xi}{\rightarrow}_{\mathcal{R}} s$. Then $\eta(t) \stackrel{\xi}{\rightarrow}_{\mathcal{R}} \eta(s)$.

Proof. The proof is immediate, since the above definitions do not depend on the name of nodes, but only on their rank w.r.t. $\succ$.

Proposition 2. Let $\mathcal{R}$ be a GRS. Let $\xi$ be an invariant $\mathcal{R}$-dependency schema. If $t$ is a term-graph, $\sigma$ is an eligible $\rho$-matcher for $t$ at a node $\alpha, \theta$ is an eligible $\pi$-matcher for $t$ at a node $\beta, s=\rho^{\sigma}[t]$. If $\beta \neq \alpha$, then $\theta \aleph_{s}^{\xi} \gamma$, for every node $\gamma \in \mathcal{N}(s)$.

Proof. Let $\xi=\left(\gg^{*}, \triangleright^{*}\right)$. Let $\rho=L \rightarrow R|\phi, \pi=G \rightarrow D| \psi$.

Assume that $\theta \bowtie_{s}^{\xi} \gamma$. By definition, we have $\beta \prec \gamma$ and there exists a node $\delta$ s.t. one of the following conditions hold: either $\delta \in \operatorname{dom}(\theta(D))$ and $\gamma \triangleright_{s}^{*} \delta$ or $\delta \in \mathcal{N}(\theta(D))$ and $\gamma \gg_{s}^{*} \delta$.

Assume that $\delta \in \operatorname{dom}(\theta(D))$ and $\gamma \triangleright_{s}^{*} \delta$. Let $\gamma^{\prime}=\gamma_{t \rightarrow s}^{-}$and $\delta^{\prime}=\delta_{t \rightarrow s}^{-}$. Since $\triangleright^{*}$ is invariant, we deduce that $\gamma^{\prime} \triangleright_{t}^{*} \delta^{\prime}$. Since $\beta$ occurs in $t, \beta_{t \rightarrow s}^{-}=\beta$.

Assume that $\sigma(R)$ redirects a node $\lambda$ occurring in $\theta(G)$. Then we have $\lambda \in$ $\operatorname{dom}(\sigma(R))$ (thus $\alpha \gg_{t}^{*} \lambda$ ). Moreover, $\lambda \in \mathcal{N}(\sigma(R))$ hence $\beta \triangleright_{t}^{*} \lambda$. We deduce that either $\theta \bowtie_{t}^{\xi} \alpha$ (if $\alpha \succ \beta$ ) or $\sigma \bowtie_{t}^{\xi} \beta$ (if $\alpha \prec \beta$ ) which is impossible since $\sigma, \theta$ are both eligible.

Consequently no node in $\theta(G)$ is redirected by $\sigma(R)$ hence we must have $\theta(G) \subseteq s$. By definition of the notion of matcher, $\delta$ must occur in $\theta(G)$. Thus $\delta=\delta^{\prime}$. We have $\beta \prec \gamma$ and $\beta \neq \alpha$ thus $\beta \prec \gamma^{\prime}$. Hence $\theta \bowtie_{t}^{\xi} \gamma^{\prime}$ which is impossible since $\theta$ is eligible.

The proof is similar if $\delta \in \mathcal{N}(\theta(D))$ and $\gamma \gg_{s}^{*} \delta$.

The next technical lemma shows that two eligible matchers necessarily commute if the dependency schema is invariant.

Lemma 2. Let $\mathcal{R}$ be a GRS and let $\xi$ be an invariant $\mathcal{R}$-dependency schema. Let $t$ be a term-graph. Assume that there exist two eligible matchers $\theta, \sigma$ for $t$ at two nodes $\alpha, \beta(\alpha \neq \beta)$ using two rules $\rho, \pi \in \mathcal{R}$ respectively.

Then $\sigma$ is an eligible $\pi$-matcher for $\rho^{\theta}[t]$ and $\theta$ is an eligible $\rho$-matcher for $\pi^{\sigma}[t]$. Moreover $\pi^{\sigma}\left[\rho^{\theta}[t]\right]=\rho^{\theta}\left[\pi^{\sigma}[t]\right]$.

Proof. Let $\rho=L \rightarrow R \mid \phi$ and $\pi=G \rightarrow D \mid \psi$. 
Assume that $\sigma$ is not a $\pi$-matcher for $\rho^{\theta}[t]$. Since $\sigma$ is a $\pi$-matcher for $t$, we have $\sigma \in \operatorname{sol}(\psi)$. Thus we must have $\sigma(G) \nsubseteq \rho^{\theta}[t]$. But we have $\sigma(G) \subseteq t$, thus $\theta(R)$ must redirect (globally or locally) a node $\gamma$ occurring in $\sigma(G)$.

But then, since $\xi$ is an $\mathcal{R}$-dependency schema, we must have $\theta \bowtie_{t}^{\xi} \beta$ (if $\beta \succ \alpha$ ) or $\sigma \rtimes_{t}^{\xi} \alpha$ (if $\alpha \succ \beta$ ) which is impossible since in this case $\alpha$ or $\beta$ would not be eligible.

Now assume that $\sigma$ is not eligible in $s=\rho^{\theta}[t]$. This means that there exists a node $\gamma$ s.t. $\sigma \bowtie_{s}^{\xi} \gamma$. By Proposition 2, we deduce that $\sigma \rtimes_{t}^{\xi} \gamma^{\prime}$ where $\gamma^{\prime}$ is the ancestor of $\gamma$, which implies that $\theta$ would not be eligible.

Therefore $\sigma$ is an eligible $\pi$-matcher for $\rho^{\theta}[t]$. By symmetry, $\theta$ is an eligible $\rho$-matcher for $\pi^{\sigma}[t]$.

Assume that there exists a node $\gamma$ occurring both in $\operatorname{dom}(\sigma(R))$ and in $\theta(D)$. Then by definition of the notion of matcher, $\gamma$ must be in $t$, thus again we have either $\theta \bowtie_{t}^{\xi} \beta$ or $\sigma \bowtie_{t}^{\xi} \alpha$ which is impossible. Thus $\operatorname{dom}(\sigma(R)) \cap \mathcal{N}(\theta(D))=\emptyset$. By symmetry, we also have $\mathcal{N}(\sigma(R)) \cap \operatorname{dom}(\theta(D))=\emptyset$.

By Proposition 1, we deduce that $(\sigma(R) ; \theta(D))[t]=(\theta(D) ; \sigma(R))[t]$.

We write $t \equiv s$ iff there exists a renaming $\eta$ for $t$ s.t. $\eta(t)=s$.

Definition 6. A rewrite system is said to be weak orthogonal if for every pair of distinct rules $\rho: L \rightarrow R \mid \phi$ and $\pi: L^{\prime} \rightarrow R^{\prime} \mid \psi$ and for every $\mathcal{N}$-mapping $\sigma \in \operatorname{sol}(\phi) \cap \operatorname{sol}\left(\phi^{\prime}\right)$ compatible with $L, L^{\prime}$ s.t. $\sigma(\operatorname{root}(L))=\sigma\left(\operatorname{root}\left(L^{\prime}\right)\right)$, we have $\sigma(R)=\eta\left(\sigma\left(R^{\prime}\right)\right)$ for some renaming $\eta$ s.t. $\eta(\alpha)=\alpha$ for every node $\alpha$ occurring in $\sigma(L)$ or $\sigma\left(L^{\prime}\right)$.

Theorem 1. (Confluence of Weak Orthogonal Systems) Let $\mathcal{R}$ be a weak orthogonal rewrite system. Let $\xi$ be an invariant $\mathcal{R}$-dependency schema. $\stackrel{\xi}{\rightarrow}_{\mathcal{R}}{ }^{*} \cup \equiv$ is confluent. Thus, if $t \stackrel{\xi}{\rightarrow}{ }^{*} \mathcal{R} s, t \breve{\succ}^{*} \mathcal{R} s^{\prime}$ and if $s, s^{\prime}$ are irreducible (w.r.t. $\stackrel{\xi}{\rightarrow} \mathcal{R}$ ), then $s \equiv s^{\prime}$.

Proof. We show that $\stackrel{\xi}{\rightarrow}_{\mathcal{R}} \cup \equiv$ is uniformly confluent, which as well known implies that $\stackrel{\xi}{\rightarrow}_{\mathcal{R}}$ is confluent.

If $t \stackrel{\xi}{\rightarrow}_{\mathcal{R}} s$ and $t \equiv t^{\prime}$, then there exists $s^{\prime} \equiv s$ s.t. $t^{\prime} \stackrel{\xi}{\rightarrow}_{\mathcal{R}} s^{\prime}$, by Lemma 1 .

If $t \equiv s$ and $t \equiv s^{\prime}$ then obviously $s \equiv s^{\prime}$.

Assume that there exists two term-graphs, $s, s^{\prime}$ s.t. $t \stackrel{\xi}{\rightarrow}_{(\rho, \sigma)} s$ and $t \stackrel{\xi}{\rightarrow}(\pi, \theta)^{\prime} s^{\prime}$. We have to show that either $s \equiv s^{\prime}$, or there exists a term-graph $t^{\prime}$ s.t. $s \stackrel{\xi}{\rightarrow} \mathcal{R} t^{\prime}$ and $s^{\prime} \stackrel{\xi}{\rightarrow}_{\mathcal{R}} t$.

Let $\rho=L \rightarrow R \mid \phi$ and $\pi=L^{\prime} \rightarrow R^{\prime} \mid \phi^{\prime}$.

If $\sigma(\operatorname{root}(L)) \neq \theta(\operatorname{root}(R))$ then the proof follows immediately from Lemma 2 . Thus we assume that $\sigma(\operatorname{root}(L))=\theta(\operatorname{root}(R))$. W.l.o.g. we assume that $\rho, \pi$ share no node. Let $\zeta=\sigma \cup \theta$. $\zeta$ is an $\mathcal{N}$-mapping compatible with $L$ and $R$. Moreover $\zeta(\operatorname{root}(L))=\zeta(\operatorname{root}(R))$. Then there exists a renaming $\eta$ s.t. $\eta(\sigma(R))=\theta\left(R^{\prime}\right)$ and $\eta$ is the identity on any node occurring in $\sigma(L), \sigma\left(L^{\prime}\right)$. W.l.o.g. we may assume that $\eta$ is the identity on $t$. Then we have $s^{\prime}=\theta\left(R^{\prime}\right)[t]=\eta(\sigma(R))[\eta(t)]=$ $\eta(\sigma(R)[t])=\eta(s)$. Hence $s \equiv s^{\prime}$. 


\section{An Example of an Invariant Dependency Schema}

From a practical point of view, we want the relation $\stackrel{\xi}{\rightarrow}_{\mathcal{R}}$ to be as weak as possible, thus the relations $\gg^{*}$ and $\triangleright^{*}$ must be as strong as possible. From a purely theoretical point of view one could take the smallest invariant relations, but of course these "ideal" relations are not computable. For instance, let $\mathcal{R}$ be a GRS containing the rules $f(\alpha: 0,0) \rightarrow \alpha: 1$ and $f(\alpha: 0,1) \rightarrow \alpha$, and let $t=\beta: f(\alpha: 0, s)$ where $s$ is an arbitrary term-graph. If $\gg^{*}$ denotes the smallest invariant dependency schema, then $\beta \gg_{t}^{*} \alpha$ iff $s \bigsqcup_{\mathcal{R}} 0$ (since the first rule is the only one that affects $\alpha$ ). Since $s$ is arbitrary, $\gg^{*}$ is non computable.

In this section, we give an example of a tractable invariant dependency schema. The provided relations are significantly stronger than the ones we proposed in [3] or in [7]. For this purpose, we need to introduce additional definitions and notations.

Let $f$ be a function symbol. An $f$-rule is a rule $L \rightarrow R \mid \phi$ s.t. $l_{L}(\operatorname{root}(L))=f$.

Let $\rho=L \rightarrow R \mid \phi$ be a rule. $\geq_{\rho}$ denotes the smallest transitive and reflexive relation containing $\geq_{L}$ s.t. if $\alpha \gg_{a} \beta \in R$ or $\alpha \gg \beta \in R$ then $\alpha \geq_{\rho} \beta$. Intuitively, $\alpha \geq \rho \beta$ states that an application of the rule $\rho$ can create a path from $\alpha$ to $\beta$.

$\geq_{\mathcal{R}}$ denotes the smallest reflexive and transitive relation s.t. $(f, a) \geq_{\mathcal{R}}(g, b)$ if $\rho=L \rightarrow R \mid \phi$ is a $f$-rule and if there exist three nodes $\alpha, \beta, \delta$ s.t. $\alpha \gg_{l} g$ and $\alpha \gg_{b} \delta$ are actions in $R, \delta \geq_{\rho} \beta$ and $a_{L}(\operatorname{root}(L)) \geq_{L} \beta$. Intuitively, this means that the function $f$ calls another function $g$ and that, moreover, a node $\beta$ initially reachable from feature $a$ of the node labeled by $f$ may become reachable from the feature $b$ of $g$.

A rule $L \rightarrow R \mid \phi$ is said to produce a side-effect on a feature a if $\operatorname{dom}(R)$ contains a node $\alpha$ occurring in $L$ but distinct from $\operatorname{root}(L)$, s.t. $a_{L}(\operatorname{root}(L)) \geq_{L} \alpha$.

A $\mathcal{F}$-family of sets of function symbols $\left(E_{a}\right)_{a \in \mathcal{F}}$ is said to be $\geq_{\mathcal{R}}$-closed iff for all $g \in E_{b}$ and for all $f$ s.t. $(f, a) \geq_{\mathcal{R}}(g, b)$, we have $f \in E_{a}$.

$\mathcal{S E}(\mathcal{R})$ is the smallest $\geq_{\mathcal{R}}$-closed $\mathcal{F}$-family of function symbols s.t. if there exists an $f$-rule producing a side-effect on $a$ then $f \in \mathcal{S E}(\mathcal{R})_{a}$.

Definition 7. We denote by $\chi$ the dependency schema defined as follows.

$\chi=\left(\gg^{*}, \triangleright^{*}\right)$, where $\gg_{t}^{*}, \triangleright_{t}^{*}$ and $\geq_{t}^{a}$ are the smallest reflexive relations s.t.:

- If $a_{t}(\alpha) \geq_{t} \beta$ and $l_{t}(\alpha) \in \mathcal{D}$ then $\alpha \geq_{t}^{a} \beta$.

- If $\alpha \geq_{t}^{a} \beta, \gamma \succ \alpha, \gamma \gg_{t}^{*} \beta$ and $\gamma \triangleright_{t}^{*} \delta$ then $\alpha \geq_{t}^{a} \delta$.

- If $\alpha \geq_{t}^{a} \beta$ for some feature $a$, then $\alpha \triangleright_{t}^{*} \beta$.

- If $l_{t}(\alpha) \in \mathcal{S E}(\mathcal{R})_{a}$ and $\alpha \geq_{t}^{a} \beta$ then $\alpha \gg_{t}^{*} \beta$.

Intuitively $\alpha \geq_{t}^{a} \beta$ expresses the fact that $\alpha$ is labeled by a defined symbol and that $\beta$ is reachable (or may become reachable after some rewriting steps) from the feature $a$ of the node $\alpha$. The first item corresponds straightforwardly to this definition. The second item is slightly more complicated and states that $\beta$ may become reachable (at some point) if there exists a node $\gamma$ that both affects a node reachable from $\alpha$ (from feature $a$ ) and depends on $\beta$. Indeed, reducing the node $\gamma$ may create a path from $\alpha$ to $\beta$. 
Using this relation, $\gg^{*}$ and $\triangleright^{*}$ are easy to define. The third item states that $\alpha$ depends on a node $\beta$ only if a path exists (or may be created) from a feature of $\alpha$ to $\beta$. The fourth item states that $\alpha$ affects $\beta$ only if $\alpha$ is labeled by a defined symbol performing a side effect on a feature $a$ and if $\beta$ is reachable (or may become reachable) from the feature $a$ of $\alpha$.

Example 4. Let $\mathcal{R}=\{f(\alpha, \beta) \rightarrow g(\beta, \alpha), g(s(\alpha), s(\beta)) \rightarrow g(\alpha, \beta), g(\alpha: 0, \beta) \rightarrow$ $\left.\beta \gg_{l} 0\right\}$. We have $(f, 1) \geq_{\mathcal{R}}(g, 2)$ and $(f, 2) \geq_{\mathcal{R}}(g, 1) . g$ performs a side effect on feature 2 (third rule) thus $g \in \mathcal{S E}(\mathcal{R})_{2}$ and $f \in \mathcal{S E}(\mathcal{R})_{1}$.

Let $t=\alpha: f\left(s(s(\beta)), s\left(s\left(\beta^{\prime}\right)\right)\right)$. We have $\alpha \gg_{t}^{*} \beta$ and $\alpha \ngtr_{t}^{*} \beta^{\prime}\left(\right.$ but $\left.\alpha \triangleright_{t}^{*} \beta^{\prime}\right)$.

Theorem 2. $\chi$ is an invariant dependency schema.

Proof. It is easy to see that $\chi$ is a dependency schema (since any node occurring in the left-hand side and distinct from the root is reachable from a given feature of the root). We prove that it is invariant.

Let $t$ be a term-graph. Let $\rho=L \rightarrow R \mid \phi$ be a rule in $\mathcal{R}$. Let $\sigma$ be a $\rho$ matcher for $t$ at a node $\alpha$. Let $s=\rho^{\sigma}[t]$. For every node $\beta$ we denote by $\beta^{\prime}$ the node $\beta_{t \rightarrow s}^{-}$.

We show by induction on the above relation that $\gg_{t}^{*}$ and $\triangleright_{t}^{*}$ are invariant, and that if $\beta \geq_{s}^{a} \delta$ then $\beta^{\prime} \geq_{t}^{b} \delta^{\prime}$ for some feature $b$ (possibly distinct from $a$ ) s.t. $\left(l_{t}\left(\beta^{\prime}\right), b\right) \geq_{\mathcal{R}}\left(l_{t}(\beta), a\right)$. According to the above inductive definitions, we need to distinguish several cases.

- If $a_{s}(\beta) \geq_{s} \delta, l_{s}(\beta) \in \mathcal{D}$.

- If both $\delta$ and $\beta$ are created by $\rho$ then the proof is obvious since $\geq_{t}^{a}$ is reflexive.

- If $\delta$ is created but not $\beta$. Since $l_{s}(\beta) \in \mathcal{D}$ the node $\beta$ cannot be locally redirected. Thus $a_{t}(\beta)=a_{s}(\beta)$ and $l_{t}(\beta)=l_{s}(\beta)$. Since $\delta$ does not occur in $t$, there exists a node $\beta^{\prime}$ s.t. $a_{t}(\beta) \geq_{t} \beta^{\prime}$ and $\beta^{\prime} \in \operatorname{dom}(\sigma(R))$.

By definition we have $\beta \geq_{t}^{a} \beta^{\prime}$ (hence $\beta \triangleright_{t}^{*} \beta^{\prime}$ ) and (since $\chi$ is a dependency schema and $\left.\alpha \gg_{t} \beta^{\prime}\right) \alpha \gg_{t}^{*} \beta^{\prime}$. Since $\alpha$ is eligible, we must have $\alpha \succ \beta$. By reflexivity $\alpha \triangleright_{t}^{*} \alpha$. Consequently, we deduce $a_{t}(\beta) \geq_{t}^{a} \alpha$ (by the second item in the definition of $\chi$ ).

- If $\beta$ is created but not $\delta$. Since $\beta \geq_{s}^{a} \delta, \sigma(R)$ contains an action of the form $\beta \gg_{a} \gamma$. We consider the last action of this form. Then $a_{s}(\beta)=\gamma$. There exists a node $\beta^{\prime}$ in $\sigma(L)$ s.t. $\beta^{\prime} \geq_{t} \delta$ and $\gamma \geq_{\sigma(\rho)} \beta^{\prime}$. $\beta^{\prime}$ must be reachable from $\alpha$ (since $\rho$ is admissible) thus there exists a feature $b$ s.t. $b_{t}(\alpha) \geq_{t} \beta^{\prime} \geq_{t} \delta$. By definition, we have $\alpha \geq_{t}^{b} \delta$. Moreover, by definition of $\geq_{\mathcal{R}},\left(l_{t}(\alpha), b\right) \geq_{\mathcal{R}}\left(l_{s}(\beta), a\right)$.

- If neither $\beta$ nor $\delta$ is created. $\rho$ cannot redirect $\beta$ since $l_{t}(\beta) \in \mathcal{D}$. $\rho$ must create a path from $a_{t}(\beta)$ to $\delta$, thus there exists two nodes $\gamma, \gamma^{\prime} \in \mathcal{N}(\sigma(L))$ s.t. $a_{t}(\beta) \geq_{t} \gamma, \gamma^{\prime} \geq_{t} \delta$ and $\gamma \in \operatorname{dom}(\sigma(R))$.

We have $\beta \triangleright_{t}^{*} \gamma$ thus $\alpha \succ \beta$. Moreover $\alpha \triangleright_{t}^{*} \delta$. Consequently, by the second point in the definition of $\geq_{t}^{a}$, we have $\beta \geq_{t}^{a} \delta$.

- If $\gamma \succ \beta, \beta \geq_{s}^{a} \lambda, \gamma \gg_{s}^{*} \lambda$ and $\gamma \triangleright_{s}^{*} \delta$. By the induction hypothesis, we have $\beta^{\prime} \geq_{t}^{b} \lambda^{\prime}$, for some feature $b$ s.t. $\left(l_{t}\left(\beta^{\prime}\right), b\right) \geq_{\mathcal{R}}\left(l_{s}(\beta), a\right)$. Moreover, we have $\gamma^{\prime} \gg_{t}^{*} \lambda^{\prime}$ and $\gamma^{\prime} \triangleright_{t}^{*} \delta^{\prime}$. Thus $\beta^{\prime} \geq_{t}^{b} \delta^{\prime}$. 
- If $\beta \triangleright_{s}^{*} \delta$, then we must have $\beta \geq_{s}^{a} \delta$ for some feature $a$. By the above property, we deduce $\beta^{\prime} \geq_{s}^{b} \delta^{\prime}$ for some feature $b$. Thus $\beta^{\prime} \triangleright_{t}^{*} \delta^{\prime}$.

- If $\beta \gg_{s}^{*} \delta$ then $l_{s}(\beta) \in \mathcal{S E}(\mathcal{R})_{a}$ and $\beta \geq_{s}^{a} \delta$. By the above property we have $\beta^{\prime} \geq_{t}^{b} \delta^{\prime}$, where $\left(l_{t}\left(\beta^{\prime}\right), b\right) \geq_{\mathcal{R}}\left(l_{s}(\beta), a\right)$. Since $\mathcal{S E}(\mathcal{R})$ is $\geq_{\mathcal{R}}$-closed we have $l_{t}\left(\beta^{\prime}\right) \in \mathcal{S E}(\mathcal{R})_{b}$. Thus $\beta^{\prime} \gg_{t}^{*} \delta^{\prime}$.

\section{$5 \quad$ Needed Rewriting}

This section is the most important part of the paper. We provide a reduction strategy which is optimal (for a particular class of GRS) in the sense that the only nodes that are reduced are needed, i.e. are reduced in every derivation ${ }^{4}$. Moreover, the length of the derivation is also minimal. This last point makes an interesting difference with term rewrite systems in which this property does not hold $^{5}$. In order to formally define these notions, we need to introduce additional definitions and notations.

Let $\mathcal{R}$ be a GRS, let $\xi$ be an $\mathcal{R}$-dependency schema. If $t$ is a term-graph and $\alpha \in \mathcal{N}(t)$, we denote by $[t]_{\alpha}^{\xi}$ the maximal term-graph s.t. $[t]_{\alpha}^{\xi} \subseteq t$ s.t. for every node $\beta \in \operatorname{dom}\left([t]_{\alpha}^{\xi}\right)$ for every $\gamma \in \mathcal{N}(t)$ s.t. $\gamma \succ \alpha$ or $l_{t}(\alpha) \notin \mathcal{D}$ we have $\gamma \ngtr_{t}^{*} \beta$.

Intuitively, $[t]_{\alpha}^{\xi}$ denotes the part of the term-graph $t$ that will surely not change until $\alpha$ is reduced. Thus if there exists a node $\gamma$ in $t$ s.t. $\gamma \gg_{t}^{*} \beta$ (i.e. " $\gamma$ possibly affects $\beta$ ") for some node $\beta \in \operatorname{dom}(s)$ then $\alpha$ must be reduced before $\gamma$, which is possible only if $\alpha$ is labeled by a defined symbol and $\alpha \succeq \gamma$. The definition is slightly different for constructor nodes (second subcondition) since in this case $\alpha$ is never reduced.

Example 5. Let $f, g$ be defined symbols and let $s, s^{\prime}, 0$ be constructors. Assume that $f$ performs side effects on feature 1 and that $g$ does not perform any side effect.

Let $t=\alpha: s\left(\alpha^{\prime}: g\left(s^{\prime}(0)\right), \beta: s^{\prime}\left(\delta: s^{\prime}(0)\right)\right) ; \gamma: g(\beta) ; \lambda: f(\delta)$. Then $[t]_{\alpha}^{\chi}=$ $\alpha: s\left(\alpha^{\prime}, s^{\prime}(\delta)\right) ; s^{\prime}(0)$. Notice that the argument of $\alpha^{\prime}$ remains in the term-graph (as a disconnected subgraph $\left.s^{\prime}(0)\right)$ but that the edges starting from $\alpha^{\prime}$ are removed. Indeed, we have $\alpha^{\prime} \gg_{t}^{*} \alpha^{\prime}$ and $\lambda \gg_{t}^{*} \delta$ (but $\gamma \gg_{t}^{*} \beta$ ).

If $t$ is a term-graph, we denote by $\hat{t}$ the term-graph obtained from $t$ by removing all the nodes that are not reachable from the root. A term-graph $t$ is said to be a value if $\hat{t}$ contains no defined symbols and if $\hat{t} \subseteq[t]_{\operatorname{root}(t)}^{\xi}$. This means that the part of the term-graph that is reachable from the root cannot

\footnotetext{
${ }^{4}$ Note that this notion of neededness implicitly assumes that rooted term-graphs are considered and that one is only interested by the part of term-graph that is reachable from the root. If the term-graphs are not rooted then obviously any node is needed (since at least it defines its own value).

5 This is due to the fact that term-graph rewrite rules do not duplicate the terms. For instance, if we apply the rule $f(x) \rightarrow g(x, x)$ on the term $f(t)$, we obtain a term like $g(\alpha: t, \alpha) . t$ is not duplicated hence it will not have to be reduced twice. It should be noticed that collapsing (i.e. the merging of identical subgraphs) is incorrect in our context.
} 
be affected by a derivation (this implies in particular that $\hat{t}$ contains no defined symbol, but this condition is not sufficient, see Example 6).

Definition 8. Let $\mathcal{R}$ be a GRS and let $\xi$ be an invariant $\mathcal{R}$-dependency schema. $A$ node $\alpha$ is said to be $\xi$-needed for a term-graph $t_{0}$ if for every sequence $t_{0}, \ldots, t_{n}$ s.t. $t_{n}$ is a value and s.t. for every $i \in[1 . . n] t_{i}=\rho_{i}^{\sigma_{i}}\left[t_{i-1}\right]$ for some rule $\rho_{i} \in \mathcal{R}$ and some eligible $\rho_{i}$-matcher $\sigma_{i}$ at a node $\beta_{i}$, there exists $i \in[1 . . n]$ s.t. $\beta_{i}=\alpha$

It should be emphasized that neededness is relative to a given dependency schema. Defining a needed rewriting strategy for the smallest possible dependency schema $\xi$ is obviously impossible, since $\xi$ is non computable as soon as side effects are possible as explain in Section 4 (even if we strongly restrict the class of GRS).

The definition of the reduction strategy is more complicated than in the usual case, because one has to handle the dependencies between the nodes. In the standard case, the only node that can affect a given node $\alpha$ is $\alpha$ itself (no side-effect). This is not the case here. Thus, a node may be needed even if it is non reachable from the root.

Example 6. Let $\mathcal{R} \stackrel{\text { def }}{=}\{\rho: g(\alpha: 0) \rightarrow \alpha: 1\}$ and $t=\delta: c(\beta: 0) ; \gamma: g(\beta)$, where $\delta$ is the root ( $c$ is a constructor). $\gamma$ is non reachable from $\delta$, but it is obviously needed for computing the normal form. The only applicable rule is $\rho$, yielding: $\delta: c(\beta: 1)$.

Similarly, a node may be needed because it "blocks" the reduction of a needed node.

Example \%. Consider the following GRS: $\mathcal{R}^{\prime} \stackrel{\text { def }}{=} \mathcal{R} \cup\left\{\rho^{\prime}: h(\alpha: 1, \beta) \rightarrow \beta ; \alpha: 0\right\}$. Let $s=\alpha: h(\gamma: 1, \beta: f(\gamma))$, where $\alpha \prec \beta$. Initially the only applicable rule is $\rho$ on $\beta$. $\rho^{\prime}$ is not applicable, for every dependency schema $\xi$ (we have $\alpha \gg_{s} \gamma$ and $\beta \triangleright_{s} \gamma$ ). Thus $\beta$ is needed, although initially only the value of $\alpha$ may seem relevant. This is mainly due to the fact that $\alpha \prec \beta$

Even if $\xi$ is computable, defining a needed strategy is not easy as illustrated by the following:

Example 8. $\mathcal{R} \stackrel{\text { def }}{=}\left\{\rho_{1}: f(s(\alpha), \beta) \rightarrow f(\alpha, \beta), \rho_{2}: f(\alpha: 0,0) \rightarrow \alpha: s(0), \rho_{3}:\right.$ $\left.f(\alpha: 0,1) \rightarrow \alpha, \pi_{1}: g(\beta: s(\alpha), 0) \rightarrow \beta: 0, \pi_{2}: g(\beta, 1) \rightarrow \beta\right\}$.

Let $t=\alpha ; \gamma: f\left(s(s(\beta: s(\alpha: 0))), s_{1}\right) ; \delta: g\left(\beta, s_{2}\right)$ (the root is $\left.\alpha\right)$. Assume that we know that $\delta \gg_{t}^{*} \alpha$. In order to decide whether $\gamma$ is $\xi$-needed or not, we have to evaluate one of the terms $s_{1}$ or $s_{2}$. If $s_{1} \rightarrow 1$ then obviously $\gamma \ngtr_{t}^{*} \alpha$ (since $\rho_{2}$ will never be applicable). Similarly, if $s_{2} \rightarrow 0$ then the link between $\gamma$ and $\alpha$ is cut by the rule $\pi_{1}$, thus $\gamma \gg_{t}^{*} \alpha$. If $s_{1} \rightarrow 0$ and $s_{2} \rightarrow 1$ then we have $\gamma \gg_{t}^{*} \alpha$. But $s_{1}, s_{2}$ are not both needed if $\gamma \ngtr_{t}^{*} \alpha$.

We could overcome this problem by imposing very strong restrictions on the considered GRS. However, we prefer to impose additional conditions on the dependency schemata, which are not too restrictive, because they are satisfied by the relations proposed in Section 4 : 
Definition 9. Let $\mathcal{R}$ be a GRS. An invariant $\mathcal{R}$-dependency schema $\xi$ is said to be strongly invariant, if for every rule $\rho=L \rightarrow R \mid \phi \in \mathcal{R}$ and for every pair of term-graphs $(t, s)$ s.t. $s=\rho^{\sigma}[t]$ for some $\rho$-matcher $\sigma$ at $\alpha$, then for every $\beta, \delta \in \mathcal{N}(t)$ s.t. $\beta \neq \alpha, \beta \gg_{t}^{*} \delta$ (resp. $\beta \triangleright_{t}^{*} \delta$ ) and $\beta \gg_{s}^{*} \delta$ (resp. $\beta \triangleright_{s}^{*} \delta$ ) we have $\alpha \gg_{t}^{*} \delta$. Moreover there exists a node $\gamma \in \operatorname{dom}(\sigma(R))$ s.t. $\beta \triangleright_{t}^{*} \gamma$.

It is easy to see that the dependency schema $\chi$ introduced in Section 4 is strongly invariant.

\subsection{Relevancy}

The definition of the strategy requires great care. For instance, one cannot assume that any node affecting the root is needed. Consider the term: $\alpha: 0 ; \beta: g(\alpha) ; \beta^{\prime}: g(\alpha)$. Then both $\beta$ and $\beta^{\prime}$ affect $\alpha$ (according to the previous definitions), but only the maximal node (according to the priority ordering) is actually needed for sure (the other one may actually be useless).

A rooted term-graph $t$ is said to be a line if $\hat{t}=t$ and if for every node $\alpha$ in $t$ there exists at most one feature $a \neq l$ s.t. $a_{t}(\alpha)$ is defined. $s$ is said to be a line of $t$ if $s$ is a line, $s \subseteq t$ and $\operatorname{root}(t)=\operatorname{root}(s)$.

Example 9. Let $t=\alpha: f(\beta: s(\gamma), \lambda: s(0))$. The term-graphs $s_{1}=\alpha: f(1 \Rightarrow \beta: s(\gamma))$ and $s_{2}=\alpha: f(2 \Rightarrow \lambda)$ are two lines of $t . s_{1}$ is maximal, $s_{2}$ is not.

If $t$ is a term-graph, $\mathcal{R}$ is a GRS, and $\xi$ is an $\mathcal{R}$-dependency schema, we denote by $\langle t\rangle_{\alpha}^{\xi}$ the maximal subgraph $s$ of $[t]_{\alpha}^{\xi}$ s.t. for every node $\beta$ of $s$ :

- Either $\alpha=\operatorname{root}(t), l_{t}(\alpha) \in \mathcal{C}$ and $\alpha \geq_{t} \beta$.

- Or there exists a rule $L \rightarrow R \mid \phi \in \mathcal{R}$, a line $l$ of $L$ and a $\mathcal{N}$-mapping $\sigma \in \operatorname{sol}(\phi)$ s.t. $\sigma(\operatorname{root}(L))=\alpha, \sigma(l) \subseteq t$ and $\beta \in \sigma(l)$.

Intuitively, $\langle t\rangle_{\alpha}^{\xi}$ denotes the part of $[t]_{\alpha}^{\xi}$ that is currently "useful" w.r.t. the GRS $\mathcal{R}$ (a node is useful either because it is reachable from the root or because it occurs in the left hand side of a rewrite rule).

Example 10. Let $t=\beta: f\left(\alpha: s\left(\alpha^{\prime}: 0\right), s\left(\alpha^{\prime}\right)\right)$. Let $\mathcal{R}=\{f(\alpha: 0, s(\beta)) \rightarrow \beta\}$. Then $\langle t\rangle_{\beta}^{\xi}=f\left(\alpha, s\left(\alpha^{\prime}\right)\right)$.

Lemma 3. Let $\mathcal{R}$ be a GRS, let $\xi$ be a strongly invariant $\mathcal{R}$-dependency schema. Let $t$ be a term-graph, let $\alpha$ be a node in $t$. Let $\sigma$ be a $\rho$-matcher for $t$ at a node $\beta$ and let $s=\rho^{\sigma}[t]$. If $\alpha \neq \beta$ then $[t]_{\alpha}^{\xi} \subseteq[s]_{\alpha}^{\xi}$.

Proof. Let $\rho=L \rightarrow R \mid \phi$. Let $t^{\prime}=[t]_{\alpha}^{\xi}$ and $s^{\prime}=[s]_{\alpha}^{\xi}$. We have to prove that $t^{\prime} \subseteq s^{\prime}$. Let $\gamma$ be a node in $t^{\prime}$.

Assume that $a_{t}(\gamma) \neq a_{s}(\gamma)$ for some feature $a$. Then we have $\gamma \in \operatorname{dom}(\sigma(R))$. This implies that $\beta \gg_{t}^{*} \gamma$. If $\beta \succ \alpha$ or if $\alpha$ is labeled by a constructor node this is impossible since $\gamma$ would not be in $\operatorname{dom}\left(t^{\prime}\right)$, by definition. Otherwise, we have $\alpha \triangleright_{t}^{*} \gamma$ and $\beta \prec \alpha$, thus $\sigma \bowtie_{t}^{\xi} \alpha$, which is impossible since $\sigma$ is eligible. Consequently, we have $t^{\prime} \subseteq s$. 
Assume that $\delta \gg_{s}^{*} \gamma$, for some node $\delta \in \mathcal{N}(s)$ s.t. $\delta \succ \alpha$. Then, since $\xi$ is invariant we deduce that $\delta_{t \rightarrow s}^{-} \gg_{t}^{*} \gamma_{t \rightarrow s}^{-}=\gamma$. We have $\delta \succ \alpha$ hence by definition $\delta_{t}^{-} \succeq \alpha$. Since $\alpha \neq \beta$ we have $\delta_{t}^{-} \succ \alpha$. Therefore $\gamma$ cannot occur in $t^{\prime}$ by definition of $[t]_{\alpha}^{\xi}$, which contradicts our assumption. Therefore, any node in $t^{\prime}$ is in $s^{\prime}$, thus $t^{\prime} \subseteq s^{\prime}$.

Let $\alpha$ be a node. We denote by $w^{\xi}(t, \alpha)$ the set of nodes $\beta$ of $t$ s.t. $\beta \gg_{t}^{*} \alpha$. $w^{\xi}(t, \alpha)$ denotes the set of nodes that possibly affect $\alpha$ ( $w$ stands for "write").

We are now in position to define our reduction strategy. To this purpose, we inductively define a set of nodes that are $\xi$-relevant in a given term-graph.

Definition 10. Let $\mathcal{R}$ be a GRS and let $\xi$ be a strongly invariant dependency schema. The set of nodes that are $\xi$-relevant in $t$ is the smallest set of nodes $\alpha$ s.t. $l_{t}(\alpha) \in \mathcal{D}$ and one of the following conditions holds:

1. Either there exist $\beta, \delta$ s.t. $\beta \prec \alpha, \beta$ is either root $(t)$ or $\xi$-relevant in $t, \delta$ is a node in $\langle t\rangle_{\beta}^{\xi}$ and $\alpha$ is the maximal node in $w^{\xi}(t, \delta)$. Intuitively, $\alpha$ is relevant, because it affects a node $\delta$ which is useful for reducing $\beta$.

2. Or $\beta$ is $\xi$-relevant, $w^{\xi}(t, \delta)=\emptyset$ for every node $\delta$ in $\langle t\rangle_{\beta}^{\xi}$ and there exists a node $\lambda$ in $\langle t\rangle_{\beta}^{\xi}$ s.t. $\beta \gg_{t} \lambda, \alpha \succ \beta$ and $\alpha \triangleright_{t}^{*} \lambda . \alpha$ is relevant because since it is of greater priority than $\beta$ and since it depends on a node $\lambda$ which is affected by $\beta$, it prevents the reduction on $\beta$ (by definition of $\stackrel{\xi}{\rightarrow}_{\mathcal{R}}$ ).

Definition 11. (Needed Reduction Strategy) Let $\mathcal{R}$ be a GRS and let $\xi$ be a strongly invariant $\mathcal{R}$-dependency schema. We write $t^{\xi} \stackrel{\xi}{\mathcal{R}}$ iff there exist a rule $\rho \in \mathcal{R}$ and an eligible $\rho$-matcher $\sigma$ for $t$ at a $\xi$-relevant node $\alpha$ s.t. $s=\rho^{\sigma}[t]$.

Proposition 3. Let $\mathcal{R}$ be a GRS and let $\xi$ be a strongly invariant $\mathcal{R}$-dependency schema. $\stackrel{\xi}{\hookrightarrow}_{\mathcal{R}} \subseteq \stackrel{\xi}{\rightarrow}_{\mathcal{R}}$.

Lemma 4. Let $\mathcal{R}$ be a GRS, let $\xi$ be a strongly invariant $\mathcal{R}$-dependency schema. Let $\rho$ be a rule in $\mathcal{R}$, let $\sigma$ be a $\rho$-matcher for $t$ at a node $\beta$ and let $s=\rho^{\sigma}[t]$. If $\beta$ is not $\xi$-relevant and $\alpha \neq \beta$ then $\langle t\rangle_{\alpha}^{\xi}=\langle s\rangle_{\alpha}^{\xi}$.

Proof. By Lemma 3 we already know that $[t]_{\alpha}^{\xi} \subseteq[s]_{\alpha}^{\xi}$. Assume that there exists a node $\delta$ in $[s]_{\alpha}^{\xi}$ not occurring in $[t]_{\alpha}^{\xi}$.

By definition, there exists a node $\lambda \succ \alpha$ s.t. $\lambda \gg_{t}^{*} \delta$. Let $\lambda$ be the maximal node having this property. Since $\beta$ is not $\xi$-relevant we have $\beta \neq \lambda$. Since $\delta \in[s]_{\alpha}^{\xi}$ we have $\lambda \ngtr_{s}^{*} \delta$. By Definition 9 this implies that $\beta \gg_{t}^{*} \delta$. Since $\delta$ is in $[s]_{\alpha}^{\xi}$, this implies that $\beta \prec \alpha$. Thus $\beta \prec \lambda$. But by Definition 9, this implies that $\sigma \bowtie_{t}^{\xi} \lambda$ which is impossible since $\sigma$ is eligible. Thus $[t]_{\alpha}^{\xi}=[s]_{\alpha}^{\xi}$, which implies that $\langle t\rangle_{\alpha}^{\xi}=\langle s\rangle_{\alpha}^{\xi}$.

Lemma 5. Let $\mathcal{R}$ be a GRS, let $\xi$ be a strongly invariant $\mathcal{R}$-dependency schema. Let $\rho$ be a rule in $\mathcal{R}$, let $\sigma$ be a $\rho$-matcher for $t$ at a node $\alpha$ and let $s=\rho^{\sigma}[t]$. If $\alpha \neq \max \left(w^{\xi}(t, \beta)\right)$ then $\max \left(w^{\xi}(t, \beta)\right)=\max \left(w^{\xi}(s, \beta)\right)$. 
Proof. Let $\gamma=\max \left(w^{\xi}(t, \beta)\right), \gamma^{\prime}=\max \left(w^{\xi}(s, \beta)\right)$. If $\gamma \neq \gamma^{\prime}$ then one of the two following conditions holds:

- Either $\gamma \notin w^{\xi}(s, \beta)$. Since $\gamma \in w^{\xi}(t, \beta)$ we have $\gamma \gg_{t}^{*} \beta$. Since $\gamma \notin w^{\xi}(s, \beta)$, we have $\gamma \gg_{s}^{*} \beta$, hence by Definition 9, this implies that $\alpha \gg_{t}^{*} \beta$, hence $\alpha \in w^{\xi}(t, \beta)$. Thus we have $\alpha \prec \gamma$. But we have $\sigma \rtimes_{t}^{\xi} \gamma$ which is impossible since $\sigma$ is eligible.

- Or we have $\gamma^{\prime} \succ \gamma, \gamma^{\prime} \gg_{s}^{*} \beta$. Since $\xi$ is invariant we have $\gamma_{t \rightarrow s}^{\prime-} \gg_{t}^{*} \beta_{t \rightarrow s}^{-}=\beta$. Since $\gamma^{\prime} \succ \gamma$ we have $\gamma_{t \rightarrow s}^{\prime-} \succ \gamma$, which is impossible by definition of $\gamma$.

Lemma 6. Let $\mathcal{R}$ be a GRS, let $\xi$ be a strongly invariant $\mathcal{R}$-dependency schema. Let $\rho$ be a rule in $\mathcal{R}$, let $\sigma$ be a $\rho$-matcher for $t$ at a node $\alpha$ and let $s=\rho^{\sigma}[t]$.

If $\alpha$ is not $\mathcal{R}$-relevant in $t$ then every node $\beta$ that is $\mathcal{R}$-relevant in $s$ is also $\mathcal{R}$-relevant in $t$.

Proof. The proof is by induction on the set of $\mathcal{R}$-relevant nodes.

1. Assume that there exists a node $\gamma$ that is either the root of $s$ or $\xi$-relevant in $s$ and a node $\delta \in \mathcal{N}\left(\langle s\rangle_{\gamma}^{\xi}\right)$ s.t. $\beta=\max \left(w^{\xi}(s, \delta)\right)$.

If $\operatorname{root}(t) \neq \operatorname{root}(s)$ then $\sigma(R)$ globally redirects the node $\operatorname{root}(t)$. If there exists a node $\gamma \succ \alpha$ s.t. $\gamma \gg_{t}^{*} \operatorname{root}(t)$, then $\sigma \bowtie_{t}^{\xi} \gamma$ hence $\sigma$ is not eligible which is impossible. Otherwise, we have $\alpha=\max \left(w^{\xi}(t, \operatorname{root}(t))\right)$, thus $\alpha$ must be $\xi$-relevant, which is impossible.

Consequently, $\gamma$ is either the root of $t$, or by the induction hypothesis, it must be $\xi$-relevant in $t$. By Lemma 4 , we have $\langle t\rangle_{\gamma}^{\xi}=\langle s\rangle_{\gamma}^{\xi}$. Since $\alpha$ is not $\mathcal{R}$ relevant, we have $\alpha \neq \max \left(w^{\xi}(t, \delta)\right)$, thus by Lemma $5, \beta=\max \left(w^{\xi}(t, \delta)\right)$. Hence $\beta$ is $\xi$-relevant.

2. Or $w^{\xi}\left(s, \delta^{\prime}\right)=\emptyset$, for all nodes $\delta^{\prime} \in \mathcal{N}\left(\langle s\rangle_{\gamma}^{\xi}\right)$, and there exist a $\xi$-relevant node $\gamma$ in $s$ and a node $\delta$ in $\langle s\rangle\rangle_{\gamma}^{\xi}$ s.t. $\gamma \gg_{s} \delta$ and $\beta \triangleright_{s}^{*} \delta$.

By the induction hypothesis, $\gamma$ is $\xi$-relevant in $t$. By Lemma 5 , for all $\delta^{\prime} \in$ $\mathcal{N}\left(\langle s\rangle_{\gamma}^{\xi}\right), w^{\xi}\left(t, \delta^{\prime}\right)$ cannot be non empty. By Lemma 4 we have $\langle t\rangle_{\gamma}^{\xi}=\langle s\rangle_{\gamma}^{\xi}$. Since $\gamma \gg_{s} \delta$ this implies that $\gamma \gg_{t} \delta$. Since $\triangleright_{t}^{*}$ is invariant, we have $\beta_{t \rightarrow s}^{-} \triangleright_{t}^{*} \delta$. If $\beta_{t \rightarrow s}^{-}=\beta$ then $\beta$ is $\xi$-relevant in $t$ thus the proof is completed. Otherwise, we must have $\alpha=\beta_{t \rightarrow s}^{-}$, thus $\alpha$ would be $\xi$-relevant, which is impossible.

The next theorem shows that $\stackrel{\xi}{\hookrightarrow} \mathcal{R}$ is normalizing, and that the length of the obtained derivations are minimal.

Theorem 3. Let $\mathcal{R}$ be a GRS. Let $t$ be a term-graph and let $\xi$ be a strongly invariant dependency schema. Assume that there exists a sequence $t_{1}, \ldots, t_{n}$ of term-graphs s.t. $t_{1}=t, t_{n}$ is a value and $t_{i} \stackrel{\xi}{\mathcal{R}}_{\mathcal{R}} t_{i+1}$, for every $i \in[1 . . n-1]$.

There exists a sequence $s_{1}, \ldots, s_{m}$ s.t. $s_{1}=t, s_{m}$ is a value, $\hat{s_{m}}=\hat{t_{n}}, m \leq n$ and $s_{i} \stackrel{\xi}{\hookrightarrow} s_{i+1}$ for every $i \in[1 . . m-1]$. 
Proof. We reason by induction on $n$. The proof is trivial for $n=0$. Otherwise, by the induction hypothesis, there exists a sequence $u_{1} \stackrel{\xi}{\hookrightarrow} \mathcal{R} \ldots \stackrel{\xi}{\hookrightarrow} \mathcal{R} u_{m}$ s.t. $u_{1}=t_{2}$, $u_{m}$ is a value and $\hat{u_{m}}=\hat{t_{n}}$.

If $t_{1} \stackrel{\xi}{\hookrightarrow} \mathcal{R} t_{2}$, then the proof is completed. Otherwise, there exists a $\rho$-matcher $\sigma$ on non $\xi$-relevant node $\alpha$ s.t. $t_{2}=\rho^{\sigma}\left[t_{1}\right]$.

Assume that $t_{2}$ is a value and $\hat{t_{2}}=\hat{t_{n}}$. Since $\alpha$ is non relevant we have $\langle t\rangle_{\operatorname{root}(t)}^{\xi}=\left\langle t_{2}\right\rangle_{\operatorname{root}(t)}^{\xi}$. Since $t_{2}$ is a value, we have $\left\langle t_{2}\right\rangle_{\operatorname{root}(t)}^{\xi}=\hat{t_{2}}=\hat{t_{n}}$. If $\hat{t} \neq\langle t\rangle_{\operatorname{root}(t)}^{\xi}$ then $\sigma(R)$ affects at least a node $\gamma$ in $\langle t\rangle_{\operatorname{root}(t)}^{\xi}$. But since $\alpha$ is eligible, this implies that $\alpha=\max \left(w^{\xi}(t, \gamma)\right)$, which is impossible since $\alpha$ is non relevant. Thus we have $\hat{t_{1}}=\hat{t_{n}}$ and the proof is obvious.

If $t_{2}$ is not a value, or if $\hat{t_{2}} \neq \hat{t_{n}}$, we must have $m>1$. There exists a $\pi$ matcher $\theta$ on a $\xi$-relevant node $\beta$ for $u_{1}$ s.t. $u_{2}=\pi^{\theta}\left[u_{1}\right]$. By Lemma $6, \beta$ is relevant in $t$. By Lemma 4 we know that $\langle t\rangle_{\beta}^{\xi}=\left\langle u_{1}\right\rangle_{\beta}^{\xi}$. Thus $\theta$ is a $\pi$-matcher for $t$ at $\beta$. Assume that $\theta$ is not eligible for $t$. There exists a node $\lambda$ s.t. $\theta \bowtie_{t}^{\xi} \lambda$. Thus there exists a node $\gamma \in \mathcal{N}\left(\langle t\rangle_{\beta}^{\xi}\right)$ s.t. either $\gamma$ is redirected by $\pi$ and $\lambda \triangleright_{t}^{*} \gamma$ or $\gamma$ is in the left-hand side of $\pi$ and $\lambda \gg_{t}^{*} \gamma$. By definition of $[t]_{\beta}^{\xi}$ we cannot have $\lambda \gg_{t}^{*} \gamma$. Thus $\lambda \triangleright_{t}^{*} \gamma$ and $\gamma$ is redirected. But since $\langle t\rangle_{\beta}^{\xi}=\left\langle u_{1}\right\rangle_{\beta}^{\xi}$, this is impossible since this would mean that $\theta$ is not eligible for $u_{1}$.

Thus $\theta$ is an eligible $\pi$-matcher for $t$ at $\beta$. By Lemma 2, $\theta$ is a $\pi$-matcher for $t_{1}, \sigma$ is a $\rho$-matcher for $u_{1}=t_{2}$ and we have $\rho^{\sigma}\left[\pi^{\theta}\left[t_{1}\right]\right]=u_{2}$.

Thus we have $t \stackrel{\xi}{\hookrightarrow} \mathcal{R} u$ where $u=\pi^{\theta}\left[t_{1}\right]$. Moreover $u \stackrel{\xi}{\rightarrow} u_{2} \stackrel{\xi}{\rightarrow}_{\mathcal{R}} \ldots \stackrel{\xi}{\rightarrow} \mathcal{R} u_{m}$, where $m \leq n-1$. By the induction hypothesis, this implies that there exists

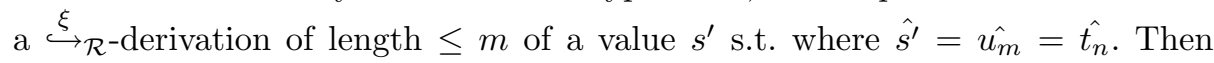
$t \stackrel{\xi}{\longrightarrow} s^{\prime}$.

\subsection{Neededness}

Now, we introduce a class of GRS for which the above strategy only reduces needed nodes.

We denote by $\mathcal{L}(t)$ the set of maximal (w.r.t. $\subseteq$ ) lines of $t$. If $\rho=L \rightarrow R$ $\phi, \pi=G \rightarrow D \mid \psi$ are two rules, we write $\rho \sqsubset \pi$ iff for every pair of lines $\left(l, l^{\prime}\right) \in(\mathcal{L}(L), \mathcal{L}(G))$ and for every $\mathcal{N}$-mapping $\sigma$ s.t. $\sigma(l) \subseteq \sigma\left(l^{\prime}\right)$, we have $\sigma(l)=\sigma\left(l^{\prime}\right)$.

A set of rules $\mathcal{R}$ is said to be elementary if for every pair of rules $\rho, \pi \in \mathcal{R}$ we have $\rho \sqsubset \pi$ (and $\pi \sqsubset \rho$ ). This condition is easy to check. Intuitively, it expresses the fact that the rules $\rho$ and $\pi$ potentially depend on the same set of nodes. For instance, the system $\{f(\alpha: s(\alpha)) \rightarrow 0, f(\alpha: s(\beta)) \rightarrow 1 \mid \alpha \neq \beta\}$ is elementary, but $\{f(\alpha: s(\beta), 0) \rightarrow 0, f(\alpha: s(s(\beta)), 1) \rightarrow 1\}$ is not.

Remark 1. The above condition may seem very restrictive. For instance, a GRS as simple as $\{f(a, s(\alpha)) \rightarrow 0, f(b, \alpha) \rightarrow 1\}$ is not elementary. However it can easily be transformed into an elementary system: $\left\{f(a, \alpha) \rightarrow f^{\prime}(\alpha), f(b, \alpha) \rightarrow\right.$ $\left.1, f^{\prime}(s(\alpha)) \rightarrow 0\right\}$. This process can be generalized: if we restrict ourselves to 
term rewrite systems, it is obvious that any inductively sequential system (i.e. any system having a definitional tree [2]) can be automatically transformed into an elementary system. Thus our results apply to any strongly/inductively sequential system $[9,10]$, since these notions coincide for constructor-based rules [8]. Actually, the notion of elementary GRS can be seen as a generalization of the notion of definitional tree (note that elementary GRS are not necessarily orthogonal).

Theorem 4. Let $\mathcal{R}$ be a GRS. Let $\xi$ be a strongly invariant $\mathcal{R}$-dependency schema. If $\mathcal{R}$ is elementary, then every $\xi$-relevant node $\beta$ for $t$ is $\xi$-needed for $t$.

Proof. The proof is by induction of the set of $\xi$-relevant nodes. Assume that there exists a derivation $t_{1} \stackrel{\xi}{\rightarrow}_{\mathcal{R}} \ldots \stackrel{\xi}{\rightarrow} \mathcal{R} t_{n}$ s.t. $t_{1}=t, t_{n}$ is a value and such that $\beta_{i} \neq \beta$, for every $i \in[1 . . n]$. We distinguish two cases, according to the inductive definition of $\xi$-relevant nodes:

- Assume that there exists a node $\beta^{\prime}$ that is either the root of $t$ or that is $\xi$-relevant in $t$ and a node $\delta \in \mathcal{N}\left(\langle t\rangle_{\beta^{\prime}}^{\xi}\right)$ s.t. $\beta=\max \left(w^{\xi}(t, \delta)\right)$.

Assume that there exists $i \in[1 . . n]$ s.t. $\beta_{i}=\beta^{\prime}$. Let $i$ be the first index having this property.

Let $s=\left[t_{i}\right]_{\beta^{\prime}}^{\xi}, s^{\prime}=\left\langle t_{i}\right\rangle_{\beta^{\prime}}^{\xi}$. By Lemma 3 , we have $[t]_{\beta^{\prime}}^{\xi} \subseteq s$. Thus $\delta$ occurs in $s$. Let $u$ be the minimal line of $s$ containing $\delta$.

By definition of $s^{\prime}$, there exists a rule $\pi=G \rightarrow D \mid \psi$ in $\mathcal{R}$ s.t. $u \subseteq \theta(G)$, for some $\mathcal{N}$-mapping $\theta$. There exists a line $v \in \mathcal{L}(G)$ s.t. $u \subseteq \theta(v)$.

Let $\rho: L \rightarrow R \mid \phi$ be the rule applied to derive $t_{i+1}$ and let $\sigma$ be the corresponding $\rho$-matcher.

By definition of the notion of $\rho$-matcher, we have $\sigma(L) \subseteq t_{i}$, thus there exists a line $v^{\prime} \in \mathcal{L}(L)$ s.t. $u \subseteq \sigma\left(v^{\prime}\right)$ or $u \supseteq \sigma\left(v^{\prime}\right)$. Let $v^{\prime}$ be the maximal sequence having this property. If $\delta$ does not occur in $\sigma\left(v^{\prime}\right)$, this implies that $\sigma\left(v^{\prime}\right) \subset u$, thus $\sigma\left(v^{\prime}\right) \subset \theta(v)$, which is impossible if $\mathcal{R}$ is elementary, since $v^{\prime}$ is maximal (we would have $\pi \not \subset \rho$ ). Therefore, $\delta$ must occur in $\sigma\left(v^{\prime}\right)$ hence in $L \sigma$.

By Lemma 5, we have $\max \left(w^{\xi}\left(t_{i}, \delta\right)\right)=\max \left(w^{\xi}(t, \delta)\right)=\beta$, which is impossible since $\beta \succ \beta^{\prime}$ by definition $\left(\beta^{\prime}=\beta_{i}\right.$ would not be eligible).

Thus there is no $i \in[1 . . n]$ s.t. $\beta_{i}=\beta^{\prime}$. This implies that $\beta^{\prime}=\operatorname{root}(t)$ by the induction hypothesis. By Lemma 3, we have $[t]_{\beta^{\prime}}^{\xi} \subseteq\left[t_{n}\right]_{\beta^{\prime}}^{\xi}$ hence $\delta$ is reachable from the root of $t_{n}$. By Lemma 5 , we have $\beta \in w^{\xi}\left(t_{n}, \delta\right)$ thus $t_{n}$ is not a value.

- Assume that there exists a node $\beta^{\prime} \xi$-relevant in $t$ and a node $\delta \in \mathcal{N}\left(\langle t\rangle_{\beta^{\prime}}^{\xi}\right)$ s.t. $w^{\xi}(t, \delta)=\emptyset, \beta \succ \delta, \beta^{\prime} \gg_{t} \delta$ and $\beta \triangleright_{t}^{*} \delta$.

By the induction hypothesis, $\beta^{\prime}$ is $\xi$-needed, thus there exists $i \in[1 . . n]$ s.t. $\beta_{i}=\beta^{\prime}$.

By Lemma $5, w^{\xi}\left(t_{j}, \delta\right)$ must be empty, for all $j \in[1 . . i]$. If $\beta \triangleright_{t_{i}}^{*} \delta$ then by Definition 9 there exists a node $\gamma$ s.t. $\beta_{j} \gg_{t_{j}}^{*} \delta$, for some $j<i$, which is impossible. Thus $\beta \triangleright_{t_{i}}^{*} \delta$.

If $\beta^{\prime} \gg_{t_{i}} \delta$, then since $\beta^{\prime} \gg_{t} \delta$, a node in $\langle t\rangle_{\beta^{\prime}}^{\xi}$ is affected during the derivation, which is again impossible.

Thus $\beta^{\prime} \gg_{t_{i}} \delta$ which is impossible since $\beta^{\prime}$ would not be eligible. 


\section{Examples}

We apply the above needed rewriting strategy on the following examples, using the dependency schema defined in Section 4. By convention, $\beta_{1} \succ \beta_{2} \succ \ldots$ Relevant nodes are marked with a box. The root is always the first mentioned node in the term-graph.

We use the rules defined in Section 3 and the following ones ${ }^{6}$ :

$$
\begin{array}{llll}
\operatorname{cadr}: \operatorname{cadr}\left(\operatorname{cons}\left(\alpha, \operatorname{cons}\left(\alpha^{\prime}, \beta\right)\right)\right) & \rightarrow \alpha^{\prime} & \operatorname{car}: \operatorname{car}(\operatorname{cons}(\alpha, \beta)) & \rightarrow \alpha \\
n f_{2}: \operatorname{nf}(s(\delta), \alpha: \operatorname{cons}(\alpha, \beta)) & \rightarrow \alpha ; n f(\delta, \beta) & n f_{1}: \operatorname{nf}(0, \alpha: \operatorname{cons}(\beta, \delta)) \rightarrow \alpha: n i l \\
+_{1}: \operatorname{plus}(s(\alpha), \beta) & \rightarrow \operatorname{plus}(\alpha, s(\beta)) & +_{2}: \operatorname{plus}(0, \beta) & \rightarrow \beta \\
\#_{1}: \operatorname{length}(\operatorname{cons}(\alpha, \beta)) & \rightarrow s(\operatorname{length}(\beta)) & \#_{2}: \operatorname{length}(n i l) & \rightarrow 0 \\
l_{0}: l_{0}(\alpha: n i l) \rightarrow \alpha: \operatorname{cons}(0, \lambda: n i l) ; l_{0}(\lambda) & &
\end{array}
$$

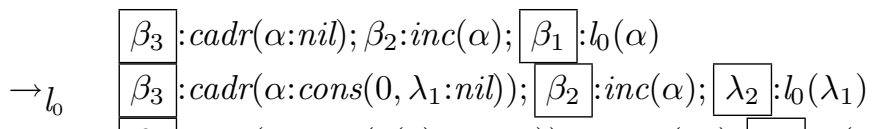

$$
\begin{aligned}
& \rightarrow i_{2} \quad \beta_{3}: \operatorname{cadr}\left(\alpha: \operatorname{cons}\left(s(0), \lambda_{1}: n i l\right)\right) ; \lambda_{3}: \operatorname{inc}\left(\lambda_{1}\right) ; \lambda_{2}: l_{0}\left(\lambda_{1}\right) \\
& \rightarrow l_{0} \quad \beta_{3}: \operatorname{cadr}\left(\alpha: \operatorname{cons}\left(s(0), \lambda_{1}: \operatorname{cons}\left(0, \lambda_{4}: n i l\right)\right)\right) ; \lambda_{3}: \operatorname{inc}\left(\lambda_{1}\right) ; \lambda_{5}: l_{0}\left(\lambda_{4}\right) \\
& \rightarrow_{i_{2}} \quad \beta_{3}: \operatorname{cadr}\left(\alpha: \operatorname{cons}\left(s(0), \lambda_{1}: \operatorname{cons}\left(s(0), \lambda_{4}: n i l\right)\right)\right) ; \lambda_{6}: \operatorname{inc}\left(\lambda_{4}\right) ; \lambda_{5}: l_{0}\left(\lambda_{4}\right) \\
& \rightarrow \text { cadr } s(0) ; \alpha: \operatorname{cons}\left(s(0), \lambda_{1}: \operatorname{cons}\left(s(0), \lambda_{4}: n i l\right)\right) ; \lambda_{6}: \operatorname{inc}\left(\lambda_{4}\right) ; \lambda_{5}: l_{0}\left(\lambda_{4}\right)
\end{aligned}
$$

At this point the normal form is known: $s(0)$. The remaining nodes are irrelevant. Note that the strict strategy diverges since the computation of $l_{0}(n i l)$ does not terminate (infinite list of 0 's). We provide a second example:

$$
\begin{aligned}
& \beta_{4}: \operatorname{plus}\left(\beta_{1}: \operatorname{car}(\delta: \operatorname{cons}(s(0), \operatorname{cons}(0, n i l))), \beta_{3}: \operatorname{length}(\delta)\right) ; \beta_{2}: n f\left(\beta_{1}, \delta\right) \\
& \rightarrow \operatorname{car} \beta_{4}: \operatorname{plus}\left(\gamma: s(0), \beta_{3}: \operatorname{length}(\delta: \operatorname{cons}(\gamma, \operatorname{cons}(0, n i l)))\right) ; \beta_{2}: n f(\gamma, \delta) \\
& \rightarrow+_{1} \quad \beta_{4}: \operatorname{plus}\left(\gamma^{\prime}: 0, s\left(\beta_{3}: \operatorname{length}\left(\delta: \operatorname{cons}\left(\gamma: s\left(\gamma^{\prime}\right), \operatorname{cons}(0, n i l)\right)\right)\right)\right) ; \beta_{2}: n f(\gamma, \delta) \\
& \rightarrow_{+2} \beta_{4}: s\left(\sqrt{\beta_{3}}: \operatorname{length}(\delta: \operatorname{cons}(\gamma: s(0), \operatorname{cons}(0, n i l)))\right) ; \beta_{2}: n f(\gamma, \delta) \\
& \rightarrow{ }_{n f_{2}} \beta_{4}: s\left(\beta_{3}: \operatorname{length}\left(\delta: \operatorname{cons}\left(\gamma: s\left(\gamma^{\prime}: 0\right), \delta^{\prime}: \operatorname{cons}(0, n i l)\right)\right) ; \lambda_{1}: n f\left(\gamma^{\prime}, \delta^{\prime}\right)\right. \\
& \rightarrow \#_{1} \beta_{4}: s\left(s\left(\lambda_{2}: \operatorname{length}\left(\delta^{\prime}: \operatorname{cons}(0, n i l)\right)\right)\right) ; \lambda_{1}: n f\left(\gamma^{\prime}, \delta^{\prime}\right) ; \delta: \operatorname{cons}\left(\gamma: s\left(\gamma^{\prime}: 0\right), \delta^{\prime}\right) \\
& \rightarrow{ }_{n f} \beta_{4}: s\left(s\left(\lambda_{2}: \operatorname{length}\left(\delta^{\prime}: n i l\right)\right)\right) ; \delta: \operatorname{cons}\left(\gamma: s\left(\gamma^{\prime}: 0\right), \delta^{\prime}\right) ; 0 ; \text { nil } \\
& \rightarrow \#_{2} \beta_{4}: s(s(0)) ; \delta^{\prime}: n i l ; \delta: \operatorname{cons}\left(\gamma: s\left(\gamma^{\prime}: 0\right), \delta^{\prime}\right) ; 0 ; \text { nil }
\end{aligned}
$$

We obtain the normal form $s(s(0))$. Notice that the first + rule is applicable although the first argument $\gamma$ occurs behind the scope of the function $n f$ because $n f$ does not perform side effects on its first argument. Thus $\beta_{2} \ngtr{ }^{*} \gamma$. This would not be the case with the dependency schema presented in [7].

${ }^{6}$ cadr, car, plus and length are standard. If $\alpha$ is a list containing at least $m$ elements, then $n f(m, \alpha)$ is the list of the $m$ first elements of $\alpha . l_{0}$ returns an infinite list of 0 's. 


\section{Conclusion}

We have presented a general framework for handling rewrite rules operating on term-graphs (encoding pointer-based data-structures), which is simpler (but similar to) the one presented in [7]. We have presented a new tractable way of detecting interferences between the defined nodes in the considered term-graph rewrite systems. Then we have provided a reduction strategy which is optimal for a class of term-graph rewrite rules, w.r.t. both the length of the derivations and the set of reduced nodes (only needed nodes are reduced). Our results extend the scope of declarative languages by allowing the programmer to define algorithms that physically affect pointer-based data-structures (as in imperative programming). Lazy reduction strategies can now be applied, similar to the ones that were already known for terms (and used by rewrite-based languages such as Haskell). From a practical side, we are now implementing a first prototype of our approach. From a more theoretical point of view, it would be interesting to extend these results to non constructor-based systems. This would require to extend the notion of strong sequentiality to GRS.

\section{References}

1. S. Antoy. Definitional trees. In H. Kirchner and G. Levi, editors, Algebraic and Logic Programming; Third International Conference; Proceedings, pages 143-157, Berlin, Germany, 1992. Springer-Verlag.

2. S. Antoy. Definitional trees. In ALP'92, volume 632 of $L N C S$, pages 143-157. Springer Verlag, 1992.

3. R. Caferra, R. Echahed, and N. Peltier. Rewriting term-graphs with priority. In Proceedings of the Eighth ACM SIGPLAN Symposium on Principles and Practice of Declarative Programming, pages 109-120. ACM Press, 2006.

4. R. Echahed and J.-C. Janodet. Admissible graph rewriting and narrowing. In Proceedings of 15th International Conference and Symposium on Logic Programming, pages 325-340, Manchester, 1998. MIT Press.

5. R. Echahed and J. C. Janodet. Parallel admissible graph rewriting. In Recent Developments in Algebraic Development Techniques, pages 121-135. LNCS 1589, 1999.

6. R. Echahed and N. Peltier. Narrowing data-structures with pointers. In Proceedings of ICGT (International Conference of Graph Transformation). Springer LNCS 4178, September 2006.

7. R. Echahed and N. Peltier. Non Strict Confluent Rewrite Systems for DataStructures with Pointers. In Proceedings of RTA (Rewriting Techniques and Applications). Springer LNCS 4533, June 2007.

8. M. Hanus, S. Lucas, and A. Middeldorp. Strongly sequential and inductively sequential term rewriting systems. Information Processing Letters, 67(1):1-8, 1998.

9. G. Huet and J.-J. Levy. Computations in orthogonal rewriting systems. In J.-L. Lassez and G. Plotkin, editors, Computational Logic: Essays in Honor of Alan Robinson, pages 395-443. MIT Press, Cambridge, MA, 1991.

10. J. W. Klop and A. Middeldorp. Sequentiality in orthogonal term rewriting systems. J. Symb. Comput., 12(2):161-195, 1991. 
11. H. Schorr and W. M. Waite. An Efficient Machine Independent Procedure for Garbage Collection in Various List Structures. Communication of the ACM, 10:501-506, August 1967.

12. S. Thompson. Haskell: The Craft of Functional Programming. Addison-Wesley, 1999. Second edition. 